\title{
One-step reduced kinetics for lean hydrogen-air deflagration
}

\author{
D. Fernández-Galisteo ${ }^{a}$, A.L. Sánchez ${ }^{a}{ }^{a}$, A. Liñán ${ }^{b}$, F.A. Williams ${ }^{c}$ \\ a Area de Mecánica de Fluidos, Univ. Carlos III de Madrid, Leganés 28911, Spain \\ b ETSI Aeronáuticos, Pl. Cardenal Cisneros 3, Madrid 28040, Spain

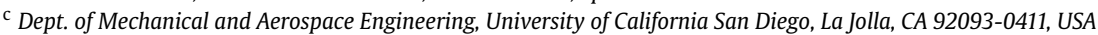

\section{A R T I C L E I N F O}

\section{Article history:}

Received 18 July 2008

Received in revised form 9 October 2008

Accepted 21 October 2008

Available online 5 November 2008

\section{Keywords:}

Hydrogen

Lean combustion

Laminar flame propagation velocity

Flammability limit

\begin{abstract}
A B S T R A C T
A short mechanism consisting of seven elementary reactions, of which only three are reversible, is shown to provide good predictions of hydrogen-air lean-flame burning velocities. This mechanism is further simplified by noting that over a range of conditions of practical interest, near the lean flammability limit all reaction intermediaries have small concentrations in the important thin reaction zone that controls the hydrogen-air laminar burning velocity and therefore follow a steady state approximation, while the main species react according to the global irreversible reaction $2 \mathrm{H}_{2}+\mathrm{O}_{2} \rightarrow 2 \mathrm{H}_{2} \mathrm{O}$. An explicit expression for the non-Arrhenius rate of this one-step overall reaction for hydrogen oxidation is derived from the seven-step detailed mechanism, for application near the flammability limit. The one-step results are used to calculate flammability limits and burning velocities of planar deflagrations. Furthermore, implications concerning radical profiles in the deflagration and reasons for the success of the approximations are clarified. It is also demonstrated that adding only two irreversible direct recombination steps to the seven-step mechanism accurately reproduces burning velocities of the full detailed mechanism for all equivalence ratios at normal atmospheric conditions and that an eight-step detailed mechanism, constructed from the seven-step mechanism by adding to it the fourth reversible shuffle reaction, improves predictions of $\mathrm{O}$ and $\mathrm{OH}$ profiles. The new reduced-chemistry descriptions can be useful for both analytical and computational studies of lean hydrogen-air flames, decreasing required computation times.
\end{abstract}

(c) 2008 The Combustion Institute. Published by Elsevier Inc. All rights reserved.

\section{Introduction}

Increased interest in the use of hydrogen has intensified needs for better understanding of its combustion behavior, for reasons of safety as well as in engine applications. Besides the necessity of being able to describe hydrogen-air ignition characteristics [1], it is especially desirable to focus on deflagrations in fuel-lean hydrogen-air mixtures, notably in hazard contexts, where release of low concentrations of hydrogen may lead to continued flame spread. As computational capabilities advance, increased use is being made of electronic computers to assess different combustion scenarios. With rare exceptions [2], full detailed hydrogen chemistry remains too complex to be used in related computational studies. Reliable reduced chemistry for lean hydrogen-air deflagrations therefore is needed for obtaining predictions computationally that can be applied ultimately for judging how to handle hydrogen in the built environment.

The hydrogen oxidation chemistry, involving only $\mathrm{H}_{2}, \mathrm{O}_{2}, \mathrm{H}_{2} \mathrm{O}$, $\mathrm{H}, \mathrm{O}, \mathrm{OH}, \mathrm{HO}_{2}$ and $\mathrm{H}_{2} \mathrm{O}_{2}$, from a global-reaction viewpoint is no

\footnotetext{
* Corresponding author. Fax: +34916249430.

E-mail address: asanchez@ing.uc3m.es (A.L. Sánchez).
}

more than a six-step mechanism, there being two atom (or element) conservation equations for the eight chemical species. In other words, although there are many more elementary chemicalkinetic reactions, there are only six independent differential equations for species conservation with nonzero chemical source terms. Various mechanisms that are reduced to fewer than six steps have been proposed and tested in the literature. These reductions evolved from pioneering investigations of steady-state and partialequilibrium approximations by Dixon-Lewis [3] and others. A fourstep mechanism with $\mathrm{H}_{2} \mathrm{O}_{2}$ and $\mathrm{HO}_{2}$ assumed to be in steady state has been found to be accurate for laminar diffusion flames, for example [4]. For fuel-lean deflagrations, a three-step mechanism has been investigated in which $\mathrm{H}_{2} \mathrm{O}_{2}$ is absent and $\mathrm{O}$ and $\mathrm{HO}_{2}$ are in steady states [5], and a two-step mechanism in which all reaction intermediates except $\mathrm{H}$ obey steady-state approximations has been shown to be reasonable [6] and has been employed to describe lean and stoichiometric hydrogen-air deflagration velocities through rate-ratio asymptotics [7].

It has long been believed that a one-step systematically reduced mechanism would be too inaccurate for any realistic application. However, it will be shown below that over a range of equivalence ratios adjacent to the lean flammability limit the concentrations of all chemical intermediates are small enough for them to follow 
Table 1

The 7-step mechanism with rate coefficients in the Arrhenius form $k=$ $A T^{n} \exp \left(-T_{a} / T\right)$ as given in [9].

\begin{tabular}{|c|c|c|c|c|}
\hline Reaction & & $A^{\mathrm{a}}$ & $n$ & $T_{a}[\mathrm{~K}]$ \\
\hline 1. $\mathrm{H}+\mathrm{O}_{2} \rightleftharpoons \mathrm{OH}+\mathrm{O}$ & & $3.52 \times 10^{16}$ & -0.7 & 8590 \\
\hline 2. $\mathrm{H}_{2}+\mathrm{O} \rightleftharpoons \mathrm{OH}+\mathrm{H}$ & & $5.06 \times 10^{4}$ & 2.67 & 3166 \\
\hline 3. $\mathrm{H}_{2}+\mathrm{OH} \rightleftharpoons \mathrm{H}_{2} \mathrm{O}+\mathrm{H}$ & & $1.17 \times 10^{9}$ & 1.3 & 1829 \\
\hline \multirow[t]{2}{*}{ 4f. $\mathrm{H}+\mathrm{O}_{2}+\mathrm{M} \rightarrow \mathrm{HO}_{2}+\mathrm{M}^{\mathrm{b}}$} & $k_{0}$ & $5.75 \times 10^{19}$ & -1.4 & 0 \\
\hline & $k_{\infty}$ & $4.65 \times 10^{12}$ & 0.44 & 0 \\
\hline 5f. $\mathrm{HO}_{2}+\mathrm{H} \rightarrow \mathrm{OH}+\mathrm{OH}$ & & $7.08 \times 10^{13}$ & 0 & 148 \\
\hline 6f. $\mathrm{HO}_{2}+\mathrm{H} \rightarrow \mathrm{H}_{2}+\mathrm{O}_{2}$ & & $1.66 \times 10^{13}$ & 0 & 414 \\
\hline 7f. $\mathrm{HO}_{2}+\mathrm{OH} \rightarrow \mathrm{H}_{2} \mathrm{O}+\mathrm{O}_{2}$ & & $2.89 \times 10^{13}$ & 0 & -250 \\
\hline
\end{tabular}

a Units are $\mathrm{mol}, \mathrm{s}, \mathrm{cm}^{3}$, and $\mathrm{K}$.

b Chaperon efficiencies are 2.5 for $\mathrm{H}_{2}, 16.0$ for $\mathrm{H}_{2} \mathrm{O}$, and 1.0 for all other species; Troe falloff with $F_{C}=0.5$ [16].

accurately a steady state approximation, while the main reactants obey the overall irreversible reaction $2 \mathrm{H}_{2}+\mathrm{O}_{2} \rightarrow 2 \mathrm{H}_{2} \mathrm{O}$, with a global hydrogen-oxidation non-Arrhenius rate determined by those of the elementary reactions of the starting detailed mechanism, shown in Table 1 . This one-step reduced mechanism is seen to provide reasonable predictions of limits for lean deflagrations as well as good results for deflagration velocities for conditions near the lean flammability limit. For richer mixtures, radical concentrations in the reaction layer increase, and their associated steady-state approximations, especially that of $\mathrm{H}$, become less accurate, leading to the failure of the one-step reduced kinetics, which away from the flammability limit must be replaced by the two-step or threestep descriptions previously derived $[5,6]$. These limitations of the one-step mechanism are explored, and the simplifications of the chemistry that lead to the one-step approximation are evaluated. The one-step result is explicit and could readily be implemented in future codes for the calculation of lean hydrogen combustion in complex configurations.

\section{Short chemistry description}

Among the different detailed hydrogen-oxygen kinetic mechanisms available in the literature, the so-called San Diego Mechanism [8] used in the following development has been tested recently and for most conditions was shown to give excellent predictions of laminar burning velocities $v_{l}$ [9], as can be seen in Fig. 1, which compares numerical results obtained with the COSILAB code [10] with three different sets of experimental data [11-13]. The computations assume adiabatic isobaric planar-flame propagation with pressure $p=1 \mathrm{~atm}$ and initial temperature $T_{u}=300 \mathrm{~K}$. The agreement between the experimental and numerical results is seen to be excellent when thermal diffusion is taken into account in the numerical description, except for very lean flames with equivalence ratio $\phi<0.4$, where the numerical integrations tend to underpredict flame velocities, independent of cross-transport effects of thermal diffusion, suggesting that premixed combustion near the lean flammability limit does not occur in the form of a uniform planar front, a result to be anticipated from concepts of cellular instabilities.

A second set of computations, now with thermal diffusion excluded, is also shown in the figure. In agreement with earlier conclusions [14], the simplified transport description produces somewhat less satisfactory results, leading to overpredictions in flame velocities on the order of $10 \%$ for stoichiometric and moderately rich mixtures. This difference is attributable to Soret diffusion of $\mathrm{H}_{2}$ out of the controlling reaction zone, towards the hot boundary, where the temperature is much higher at these near-stoichiometric conditions. For the fuel-lean mixtures of interest here, however, the temperature of the controlling reaction zone is not very different from the maximum temperature, so that the Soret effect is much less important for planar conditions, and it is seen in Fig. 1

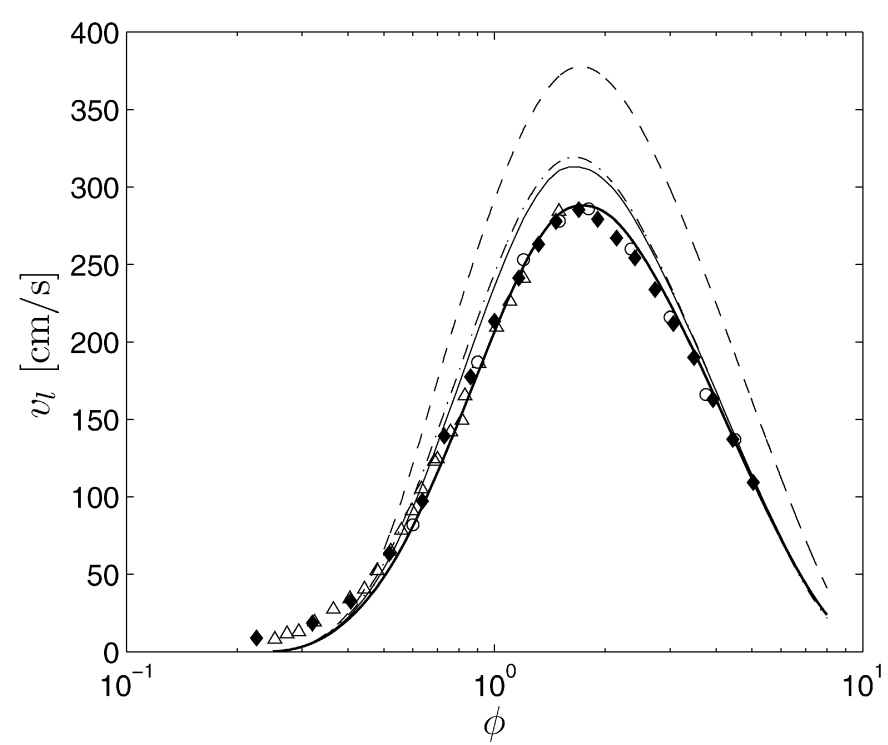

Fig. 1. The variation with equivalence ratio of the propagation velocity of premixed hydrogen-air flames for $p=1 \mathrm{~atm}$ and $T_{u}=300 \mathrm{~K}$ as obtained from experiments ([11]: diamonds; [12]: triangles; [13]: circles), from numerical integrations with the detailed chemistry and thermal diffusion included (thick solid curve) and with thermal diffusion excluded (detailed mechanism: thin solid curve; 9-step short mechanism: dot-dashed curve; 7-step short mechanism: dashed curve).

that the resulting differences become negligible for lean flames. Since it is possible to focus most directly on the chemistry by excluding transport complexities, thermal diffusion will be omitted in the following development, and therefore the numerical results represented by the thin solid line in Fig. 1 will be taken as the basis for comparison with those to be obtained below. Since effects of nonplanar diffusion will not be investigated here, the lean-flame experimental results will not be considered further; they are, however, addressed elsewhere [15].

The San Diego Mechanism [8], of 21 reversible steps, is simplified further by noticing that, for hydrogen-oxygen systems, nine elementary reactions, only three of which are reversible, suffice to describe accurately hydrogen-air laminar burning velocities over the whole range of flammability conditions at pressures sufficiently below the third explosion limit of the hydrogen-oxygen system. This short mechanism includes the seven reactions shown in Table 1 , together with the recombination reactions $\mathrm{H}+\mathrm{H}+\mathrm{M} \rightarrow$ $\mathrm{H}_{2}+\mathrm{M}$ and $\mathrm{H}+\mathrm{OH}+\mathrm{M} \rightarrow \mathrm{H}_{2} \mathrm{O}+\mathrm{M}$, which become important for sufficiently rich mixtures, where the high temperatures lead to large radical concentrations, promoting two-radical reactions. Flame velocities computed with these 9 elementary reactions with thermal diffusion neglected are also included in Fig. 1, showing excellent agreement with the detailed-chemistry computations.

For mixtures that are very fuel lean, of interest in the present analysis, radical concentrations take on very small values, causing the direct recombination reactions $\mathrm{H}+\mathrm{H}+\mathrm{M} \rightarrow \mathrm{H}_{2}+$ $\mathrm{M}$ and $\mathrm{H}+\mathrm{OH}+\mathrm{M} \rightarrow \mathrm{H}_{2} \mathrm{O}+\mathrm{M}$, which require three-body collisions involving two radicals, to become very slow compared with reaction $4 \mathrm{f}$ of Table 1 [7]. The chemistry description reduces then to the seven steps shown in Table 1, which include the three reversible shuffle reactions $1-3$, the irreversible recombination reaction $4 \mathrm{f}$, and the three irreversible $\mathrm{HO}_{2}$-consuming reactions $5 \mathrm{f}-7 \mathrm{f}$. The table shows the rate constants for all reactions, determining their dependence on the temperature $T$, except for the reverse of the shuffle reactions, whose rate constants must be obtained from the corresponding equilibrium constants. In calculating the pressure dependence of the reaction-rate constant $k_{4 f}=$ $F k_{0} /\left(1+k_{0} C_{\mathrm{M}} / k_{\infty}\right)$ we have evaluated the falloff factor $F$ from the general expression derived in [16] and present in [10] and in 


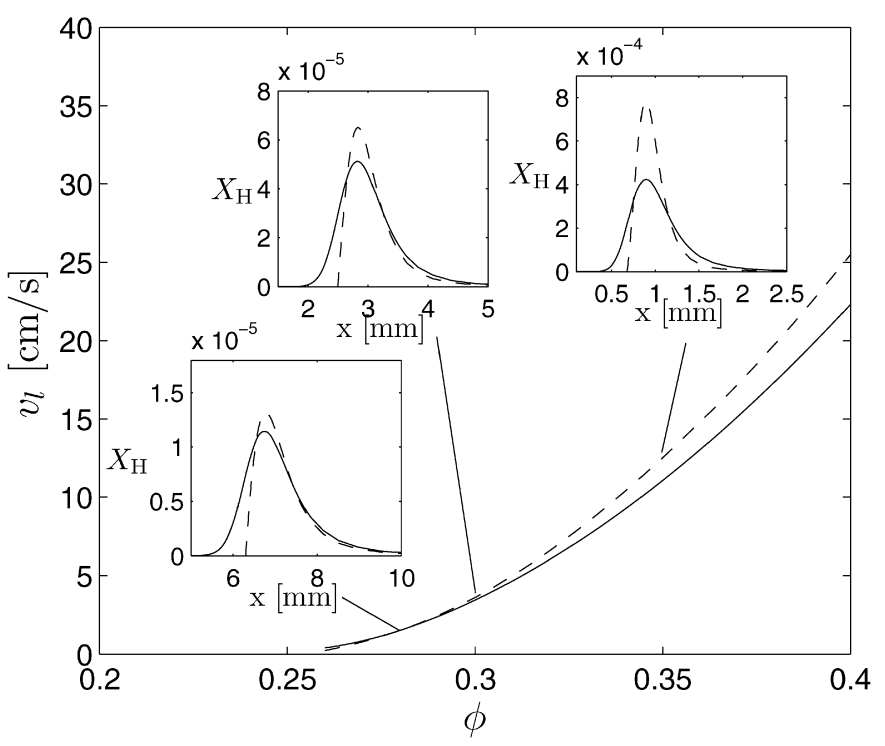

Fig. 2. The variation with equivalence ratio of the $\mathrm{H}_{2}$-air flame-propagation velocity $v_{l}$, as obtained from numerical computations with detailed chemistry (solid curve) and with the 7-step mechanism (dashed curve) for $p=1 \mathrm{~atm}$ and $T_{u}=300 \mathrm{~K}$. The insets compare the H-atom mol-fraction profiles in the flame for the 7-step computations (solid curves) with those obtained by evaluating Eq. (27) for $\phi=$ $(0.28,0.3,0.35)$ (dashed curves).

other available codes, which in the range of pressures investigated gives values that differ only by a small amount from those computed with the simpler expression $F=(0.5)^{\left\{1+\left[0.8 \log \left(k_{0} C_{M} / k_{\infty}\right)\right]^{2}\right\}^{-1}}$ proposed more recently in [17] for reaction $4 \mathrm{f}$, but not included in most codes, although new developments are now making this available in [10]. Although, like direct recombination, this step $4 \mathrm{f}$ might be thought to be in the low-pressure limit under normal conditions, falloff was found computationally to be not entirely negligible for it even at $p=1 \mathrm{~atm}, T_{u}=300 \mathrm{~K}$.

Results of flame computations with the 7-step mechanism of Table 1 are represented by the dashed curve in Fig. 1. As can be seen, the 7-step mechanism tends to overpredict flame propagation velocities, with errors that are of the order of $20 \%$ for $\phi=0.6$ and that become even larger for stoichiometric and rich flames. The performance of the 7-step mechanism in very fuel-lean conditions is tested further in Fig. 2, which shows a blowup of Fig. 1 with comparisons of the detailed and 7-step chemistry computations, along with $\mathrm{H}$-atom profiles, to be discussed later. Clearly, the errors in $v_{l}$ are reasonably small, thereby justifying the adoption of the 7-step short mechanism as the starting point of the reducedchemistry analysis.

It should be noted that this simplified chemical-kinetic mechanism leads to a flame velocity that tends to zero as a kinetically determined lean flammability limit is approached. This flammability limit is however not observed in computations of planar adiabatic flames if the $\mathrm{H}_{2} \mathrm{O}_{2}$ chemistry is included, when a slow deflagration, with a propagation velocity on the order of a few $\mathrm{mm} / \mathrm{s}$ at atmospheric conditions, is obtained for very lean mixtures beyond the kinetically determined lean flammability limit of the 7-step mechanism. In reality, such slow flames would readily extinguish in the presence of the slightest heat loss, so that their relevance for practical purposes is very limited, except at sufficiently high pressure, when the associated propagation velocity becomes significant, as discussed below in Section 6 .

To investigate the accuracy with which the 7-step mechanism describes the radical pool, Figs. 3 and 4 show profiles of radical mol fractions $X_{i}\left(i=\mathrm{H}, \mathrm{OH}, \mathrm{O}\right.$ and $\left.\mathrm{HO}_{2}\right)$ across the flame for $\phi=$ 0.3 and $\phi=0.5$, respectively. The $\mathrm{H}_{2}$ mol fraction is also shown in the upper plots (it is essentially the same in the lower plots)
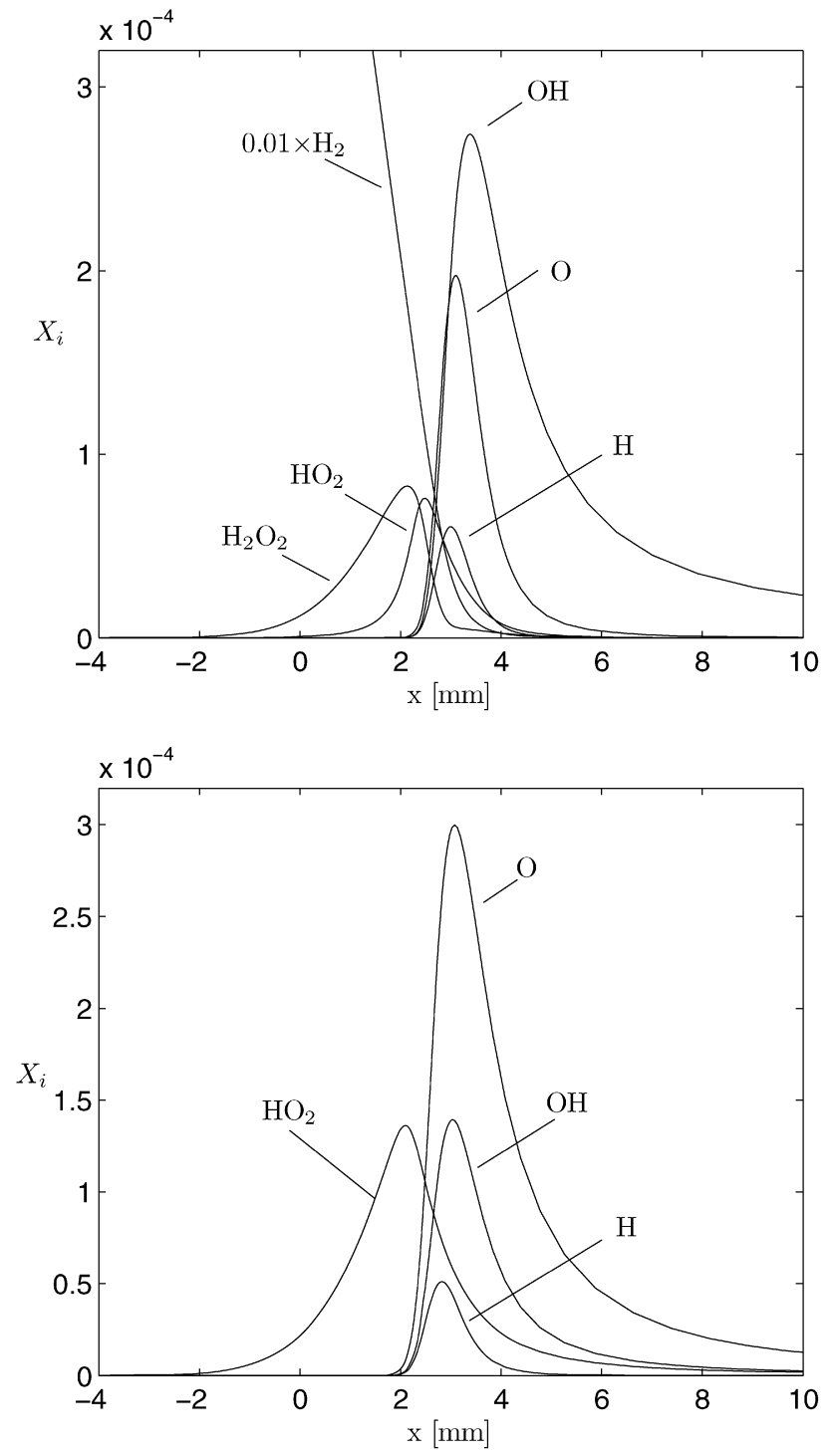

Fig. 3. Profiles of the radical mol fractions in the flame as obtained from detailed kinetics (upper plot) and from the 7-step mechanism (lower plot) for $\phi=0.3, p=$ $1 \mathrm{~atm}$ and $T_{u}=300 \mathrm{~K}$.

to enable comparisons of its magnitude with that of the radicals to be made. Also, the profile of $\mathrm{H}_{2} \mathrm{O}_{2}$ is included in the detailedchemistry results to help clarify the following interpretations.

It can be seen from Figs. 3 and 4 that the resulting $\mathrm{H}$-atom mol fraction compares reasonably well with that obtained from detailed-chemistry computations for both initial compositions. The comparison is more favorable for $\phi=0.3$, whereas for $\phi=0.5$ the 7-step description tends to overpredict $X_{\mathrm{H}}$, mainly because of the neglect of the recombination reaction $\mathrm{H}+\mathrm{OH}+\mathrm{M} \rightarrow \mathrm{H}_{2} \mathrm{O}+\mathrm{M}$ and, to a lesser extent, $\mathrm{H}+\mathrm{H}+\mathrm{M} \rightarrow \mathrm{H}_{2}+\mathrm{M}$.

On the other hand, the $\mathrm{HO}_{2}$ mol fraction is noticeably different for the 7-step mechanism at the lowest equivalence ratio. The $\mathrm{HO}_{2}$ concentration is relatively small for $\phi=0.5$ but reaches values comparable to those of the other radicals for $\phi=0.3$. The peak value of $\mathrm{X}_{\mathrm{HO}_{2}}$ is located approximately at the position where $\mathrm{H}, \mathrm{O}$ and $\mathrm{OH}$ vanish. The 7-step chemistry tends to overpredict $X_{\mathrm{HO}_{2}}$ both at the peak and also farther upstream. This discrepancy is explained by the fact that the 7-step description considers only the $\mathrm{HO}_{2}$ consumption reactions $5 \mathrm{f}-7 \mathrm{f}$, which involve hydroperoxyl collisions with either $\mathrm{H}$ or $\mathrm{OH}$, but does not include the hydroperoxyl recombination reaction $\mathrm{HO}_{2}+\mathrm{HO}_{2} \rightarrow \mathrm{H}_{2} \mathrm{O}_{2}+\mathrm{O}_{2}$. This latter 

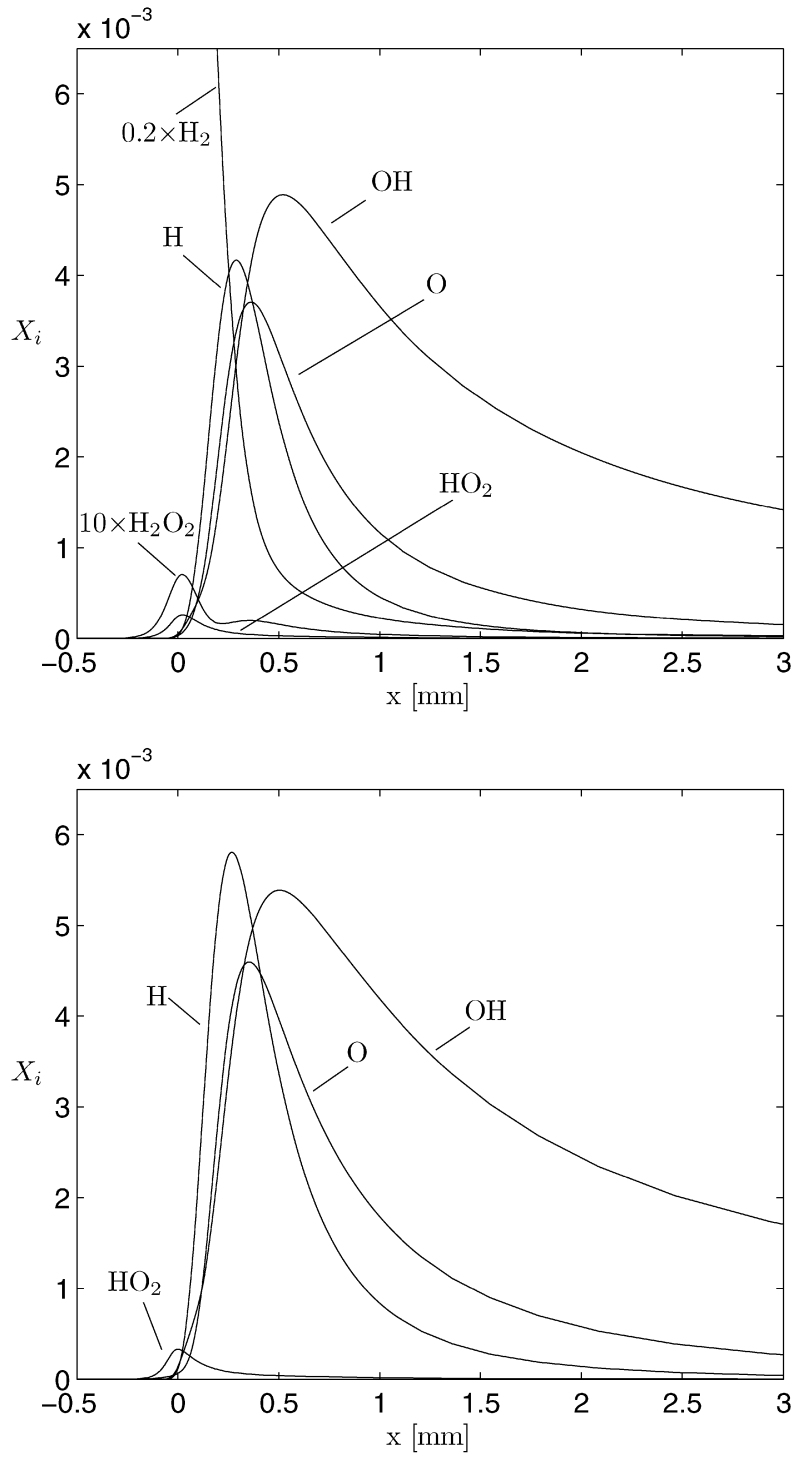

Fig. 4. Profiles of the radical mol fractions in the flame as obtained from detailed kinetics (upper plot) and from the 7-step mechanism (lower plot) for $\phi=0.5, p=$ $1 \mathrm{~atm}$ and $T_{u}=300 \mathrm{~K}$.

reaction becomes the dominant $\mathrm{HO}_{2}$ consumption reaction in the absence of $\mathrm{H}$ and $\mathrm{OH}$, and it is responsible for the appearance of significant amounts of $\mathrm{H}_{2} \mathrm{O}_{2}$, at the expense of a relatively rapid decay of $\mathrm{HO}_{2}$, upstream from the location of $\mathrm{H}$ and $\mathrm{OH}$ depletion, as can be observed in the upper plot of Fig. 3. Consideration of the hydroperoxyl recombination reaction $\mathrm{HO}_{2}+\mathrm{HO}_{2} \rightarrow \mathrm{H}_{2} \mathrm{O}_{2}+\mathrm{O}_{2}$ is therefore needed in this upstream region for an accurate description of $\mathrm{HO}_{2}$; the sum of $\mathrm{HO}_{2}$ and $\mathrm{H}_{2} \mathrm{O}_{2}$ mole fraction calculated with the full mechanism approximates the $\mathrm{HO}_{2}$ mole fraction of the 7-step mechanism fairly closely. Description of $\mathrm{H}_{2} \mathrm{O}_{2}$ production is, however, unnecessary for the computation of the overall combustion rate at pressures far enough below the third explosion limit, as seen below, and it will not be introduced here.

Figs. 3 and 4 also reveal that the 7-step description for $X_{0}$ and $X_{\mathrm{OH}}$, which is reasonable for $\phi=0.5$, is much less satisfactory for $\phi=0.3$, where the 7-step mechanism gives too large a concentration of $\mathrm{O}$ atoms and too small a concentration of $\mathrm{OH}$, which decays downstream much too fast. This disagreement can be remedied by including in the chemistry the fourth shuffle reaction $\mathrm{H}_{2} \mathrm{O}+\mathrm{O} \stackrel{8}{\rightleftharpoons} \mathrm{OH}+\mathrm{OH}$. For sufficiently rich conditions (e.g., $\phi \gtrsim 0.4$ for $p=1 \mathrm{~atm}$ and $T_{u}=300 \mathrm{~K}$ ) this reaction maintains partial equilibrium throughout the controlling reaction zone and need not be taken into account in the computation; consideration of the shuffle reactions 1-3 suffices to describe accurately $X_{0}$ and $X_{\mathrm{OH}}$ in the presence of this partial equilibrium. For leaner flames, however, reaction 8 is no longer in partial equilibrium and needs to be included in the chemistry description for a correct computation of the $\mathrm{OH}$ and $\mathrm{O}$ content of the radical pool, a point that is investigated further in Appendix A.

In any case, inclusion of the reaction $\mathrm{HO}_{2}+\mathrm{HO}_{2} \rightarrow \mathrm{H}_{2} \mathrm{O}_{2}+$ $\mathrm{O}_{2}$, necessary for a correct description of the $\mathrm{HO}_{2}$ profile upstream from the location of $\mathrm{H}$ and $\mathrm{OH}$ depletion, and of the reaction $\mathrm{H}_{2} \mathrm{O}+\mathrm{O} \stackrel{8}{\rightleftharpoons} \mathrm{OH}+\mathrm{OH}$, necessary to describe the profiles of $\mathrm{O}$ and $\mathrm{OH}$ for lean flames near the flammability conditions, does not modify appreciably the resulting $\mathrm{H}$-atom profile, which is described with sufficient accuracy by the 7-step mechanism for both $\phi=0.3$ and $\phi=0.5$. As seen below, it is the concentration of $H$ that determines the burning rate for very lean conditions, and therefore the following analysis will use the 7-step mechanism as a starting point for the reduced-chemistry development, the objective being the derivation of a one-step mechanism that correctly predicts flame propagation velocities. The augmented mechanism that follows from adding reaction 8 , necessary to compute $\mathrm{O}$ and $\mathrm{OH}$ concentrations accurately, is analyzed separately in Appendix A.

\section{One-step reduced kinetics}

Previous investigators of hydrogen-air combustion have simplified the chemistry by assuming that $\mathrm{O}, \mathrm{OH}$ and $\mathrm{HO}_{2}$ maintain steady states throughout the flame, so that $\mathrm{H}$ remains the only intermediate species not following a steady-state approximation [6]. The chemistry description is then simplified to a well-known twostep reduced mechanism composed of an overall branching reaction $3 \mathrm{H}_{2}+\mathrm{O}_{2} \rightleftharpoons 2 \mathrm{H}+2 \mathrm{H}_{2} \mathrm{O}$, with a rate given mainly by that of the elementary reaction $\mathrm{H}+\mathrm{O}_{2} \stackrel{1 f}{\rightleftharpoons} \mathrm{OH}+\mathrm{O}$, and an overall recombination reaction $2 \mathrm{H} \rightarrow \mathrm{H}_{2}$, with a rate given mainly by that of the elementary reaction $\mathrm{H}+\mathrm{O}_{2}+\mathrm{M} \stackrel{4 f}{\rightarrow} \mathrm{HO}_{2}+\mathrm{M}$. This simple mechanism, used in analytical developments [7], was found to provide predictions of flame structure and propagation velocities in good agreement with those obtained with detailed chemistry [6]. If $\mathrm{H}$ is also put into steady state, then this mechanism becomes a one-step mechanism. Previous efforts to accomplish this have not produced satisfactory results, primarily because of further approximations that were introduced to make the one-step reaction-rate description tractable. For example, step $7 \mathrm{f}$ was omitted in certain steady-state formulas in [7]. Such "truncation" approximations are not made here; the present one-step kinetics can be viewed as being derivable from the two-step description by introducing the $\mathrm{H}$-atom steady state while fully retaining all of the elementary steps of Table 1.

For the conditions of interest here, fuel-lean mixtures not too far from the flammability limit, the concentrations of all four radicals $\mathrm{H}, \mathrm{O}, \mathrm{OH}$ and $\mathrm{HO}_{2}$ are so small that they can be assumed to be in steady state, although the accuracy of the approximation decreases for richer flames. To illustrate this, we have plotted in Fig. 5 the variation of the rates of chemical production, chemical consumption and transport of the four radicals as obtained from the detailed-chemistry computations for $\phi=0.3$ and $\phi=0.5$. It can be seen that for $\phi=0.3$ the radical concentrations, shown in Fig. 3, are so small that their resulting transport rates are negligible compared with their chemical rates everywhere across the reaction zone for all four radicals. For $\phi=0.5$, the concentrations of $\mathrm{O}, \mathrm{OH}$ and $\mathrm{H}$ are much larger, as can be seen in Fig. 4, while that of $\mathrm{HO}_{2}$ remains comparatively small. The corresponding transport rates of $\mathrm{O}, \mathrm{OH}$ and $\mathrm{HO}_{2}$ are still negligible, as can be seen in Fig. 5. Although $\mathrm{H}$ appears in concentrations that are comparable to those 
$\phi=0.3:$
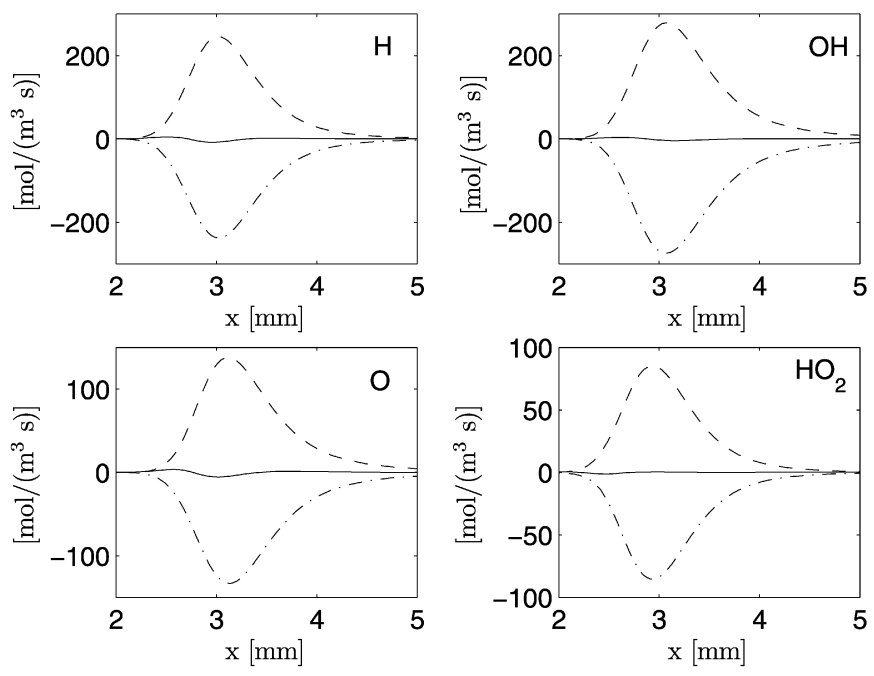

$\phi=0.5$ :
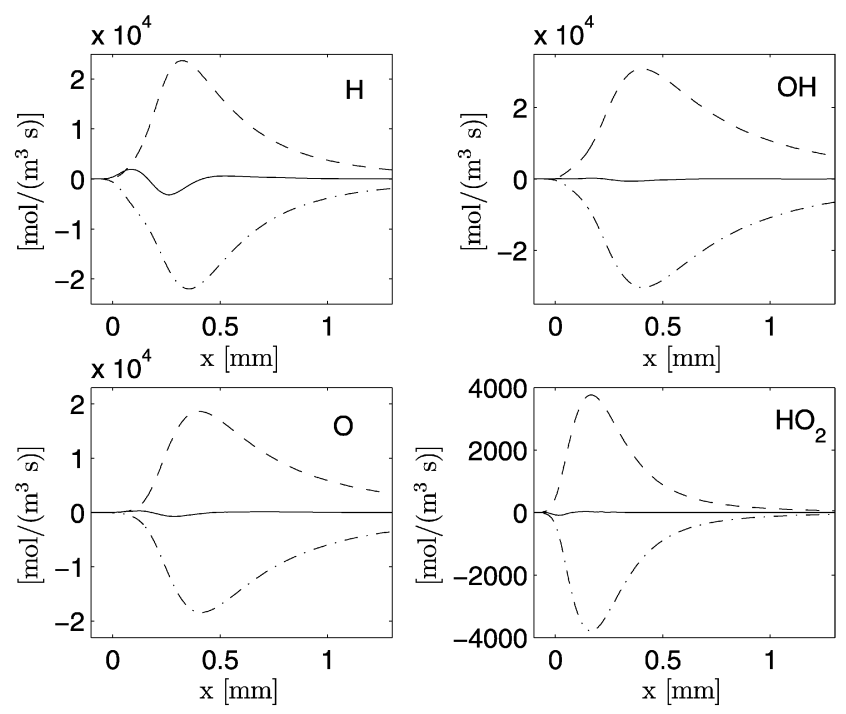

Fig. 5. The rates of production (dashed curves), consumption (dot-dashed curves) and transport (solid curves) for $\mathrm{H}, \mathrm{OH}, \mathrm{O}$ and $\mathrm{HO}_{2}$ across the flame as obtained with detailed chemistry for $p=1$ atm and $T_{u}=300 \mathrm{~K}$.

of $\mathrm{O}$ and $\mathrm{OH}$, its diffusivity is about five times larger, leading to a transport rate that can be seen in Fig. 5 to be comparable to the $\mathrm{H}$-atom chemical rates in the upstream part of the reaction zone, in agreement with previous results [6]. In view of Fig. 5, one can expect the steady-state approximation for all four intermediates to provide a very accurate description for $\phi=0.3$ and less accurate results for $\phi=0.5$. This situation is different from that encountered in autoignition, in which $\mathrm{HO}_{2}$ is not in steady state, $\mathrm{OH}$ and $\mathrm{O}$ obey good steady states only under fuel-rich conditions, and the $\mathrm{H}$ steady state is accurate only for $\phi \lesssim 0.05$ [18].

To begin to incorporate the steady-state approximations in the chemistry description, the production rates associated with the 7step mechanism are first written in general as

$\dot{C}_{0}=\omega_{1}-\omega_{2}$,

$\dot{C}_{\mathrm{OH}}=\omega_{1}+\omega_{2}-\omega_{3}+2 \omega_{5 f}-\omega_{7 f}$,

$\dot{C}_{\mathrm{H}}=-\omega_{1}+\omega_{2}+\omega_{3}-\omega_{4 f}-\omega_{5 f}-\omega_{6 f}$,
$\dot{C}_{\mathrm{HO}_{2}}=\omega_{4 f}-\omega_{5 f}-\omega_{6 f}-\omega_{7 f}$,

$\dot{\mathrm{C}}_{\mathrm{H}_{2}}=-\omega_{2}-\omega_{3}+\omega_{6 f}$,

$\dot{C}_{\mathrm{O}_{2}}=-\omega_{1}-\omega_{4 f}+\omega_{6 f}+\omega_{7 f}$,

$\dot{\mathrm{C}}_{\mathrm{H}_{2} \mathrm{O}}=\omega_{3}+\omega_{7 f}$,

where $\omega_{j}$ is the rate of reaction $j$ and $\dot{C}_{i}$ is the production rate of species $i$ (mol per unit time per unit volume), with $C_{i}$ denoting below the concentration of species $i$. Use of linear combinations of the above expressions leads to

$\dot{C}_{\mathrm{H}_{2}}+\left\{\dot{C}_{\mathrm{O}}+\frac{1}{2} \dot{C}_{\mathrm{OH}}+\frac{3}{2} \dot{C}_{\mathrm{H}}-\frac{1}{2} \dot{C}_{\mathrm{HO}_{2}}\right\}=-2 \omega_{4 f}$,

$\dot{C}_{\mathrm{O}_{2}}+\left\{\dot{C}_{\mathrm{O}}+\frac{1}{2} \dot{C}_{\mathrm{OH}}+\frac{1}{2} \dot{C}_{\mathrm{H}}+\frac{1}{2} \dot{C}_{\mathrm{HO}_{2}}\right\}=-\omega_{4 f}$,

$\dot{C}_{\mathrm{H}_{2} \mathrm{O}}-\left\{\dot{C}_{\mathrm{O}}+\dot{C}_{\mathrm{H}}-\dot{C}_{\mathrm{HO}_{2}}\right\}=2 \omega_{4 f}$,

as replacements for Eqs. (5)-(7). At steady state, radicals can be anticipated to achieve concentrations that are much smaller than those of the reactants and $\mathrm{H}_{2} \mathrm{O}$, so that the terms in curly brackets can be discarded in (8)-(10). The resulting expressions

$$
-\frac{1}{2} \dot{C}_{\mathrm{H}_{2}}=-\dot{C}_{\mathrm{O}_{2}}=\frac{1}{2} \dot{C}_{\mathrm{H}_{2} \mathrm{O}}=\omega_{4 f}=k_{4 f} C_{\mathrm{M}} C_{\mathrm{O}_{2}} C_{\mathrm{H}}
$$

indicate that, because of the steady-state approximations for the radicals, the 7-step short mechanism reduces to the global reaction

$2 \mathrm{H}_{2}+\mathrm{O}_{2} \rightarrow 2 \mathrm{H}_{2} \mathrm{O}$

with a rate equal to that of reaction $4 \mathrm{f}$. In view of the chaperon efficiencies listed in Table $1, C_{\mathrm{M}}=\left(1+15 X_{\mathrm{H}_{2} \mathrm{O}}+1.5 X_{\mathrm{H}_{2}}\right) p /\left(R^{0} T\right)$, where $X_{i}$ denotes the mole fraction of species $i$, and $R^{0}$ is the universal gas constant.

\section{Steady-state expressions for the radical concentrations}

To determine the concentrations of the radicals, in particular that of $\mathrm{H}$ atoms, which is needed for the computation of $\omega_{4 f}$, it is necessary to use the algebraic steady-state equations,

$\omega_{1}-\omega_{2}=0$,

$\omega_{1}+\omega_{2}-\omega_{3}+2 \omega_{5 f}-\omega_{7 f}=0$,

$-\omega_{1}+\omega_{2}+\omega_{3}-\omega_{4 f}-\omega_{5 f}-\omega_{6 f}=0$,

$\omega_{4 f}-\omega_{5 f}-\omega_{6 f}-\omega_{7 f}=0$,

obtained from (1)-(4), leading to exact explicit expressions for all four radicals in terms of the concentrations of $\mathrm{O}_{2}, \mathrm{H}_{2}, \mathrm{H}_{2} \mathrm{O}$ and the temperature. The development starts by employing (13) and (16), respectively, to write

$\frac{C_{\mathrm{O}}}{C_{\mathrm{H}}}=\frac{k_{1 f} C_{\mathrm{O}_{2}}+k_{2 b} C_{\mathrm{OH}}}{k_{1 b} C_{\mathrm{OH}}+k_{2 f} C_{\mathrm{H}_{2}}}$

and

$\frac{C_{\mathrm{HO}_{2}}}{C_{\mathrm{H}}}=\frac{k_{4 f} C_{\mathrm{M}} C_{\mathrm{O}_{2}}}{\left(k_{5 f}+k_{6 f}\right) C_{\mathrm{H}}+k_{7 f} C_{\mathrm{OH}}}$.

On the other hand, adding (13) and (15) and solving for $C_{\mathrm{OH}} / C_{\mathrm{H}}$ provides

$\frac{C_{\mathrm{OH}}}{C_{\mathrm{H}}}=\frac{G k_{4 f} C_{\mathrm{M}} C_{\mathrm{O}_{2}}}{k_{3 f} C_{\mathrm{H}_{2}}}$,

where

$G=\frac{1+\gamma_{3 b}}{2}+\frac{f}{2}\left\{\left[1+2\left(3+\gamma_{3 b}\right) / f+\left(1+\gamma_{3 b}\right)^{2} / f^{2}\right]^{1 / 2}-1\right\}$ 
is a function of the rescaled fuel concentration

$f=\frac{k_{5 f}+k_{6 f}}{k_{7 f}} \frac{k_{3 f}}{k_{4 f} C_{\mathrm{M}}} \frac{C_{\mathrm{H}_{2}}}{C_{\mathrm{O}_{2}}}$,

with

$\gamma_{3 b}=\frac{k_{3 b} C_{\mathrm{H}_{2} \mathrm{O}}}{k_{4 f} C_{\mathrm{M}} C_{\mathrm{O}_{2}}}$

representing the ratio of the rates of reactions $3 \mathrm{~b}$ and $4 \mathrm{f}$. The function $G$ is of order unity and approaches the limiting values $G=1+\gamma_{3 b}$ for $f \ll 1$ and $G=2+\gamma_{3 b}$ for $f \gg 1$.

Adding now (14), (15) and (16) and using (17), (18) and (19) yields an equation that can be solved for $\mathrm{C}_{\mathrm{OH}}$ to give

$C_{\mathrm{OH}}=\frac{1}{H} \frac{k_{2 f} C_{\mathrm{H}_{2}}}{k_{1 b}}\left(\frac{k_{1 f}}{k_{4 f} C_{\mathrm{M}}} \frac{f+G}{\alpha f+G}-1\right)$,

where

$H=\frac{1}{2}+\frac{1}{2}\left[1+4 \gamma_{2 b} f \frac{f+G}{\alpha f+G}\left(\frac{k_{1 f}}{k_{4 f} C_{M}} \frac{f+G}{\alpha f+G}-1\right)\right]^{1 / 2}$

with

$\gamma_{2 b}=\frac{k_{7 f}}{k_{5 f}+k_{6 f}} \frac{k_{2 b} k_{2 f}}{k_{1 b} k_{3 f}}$

and

$\alpha=\frac{k_{6 f}}{k_{5 f}+k_{6 f}}$.

Evaluation of these reaction-rate ratios indicates that $\gamma_{2 b} \ll 1$ in the range of temperatures of interest (e.g., $\gamma_{2 b} \simeq 1.5 \times 10^{-3}$ at $T=$ $1000 \mathrm{~K}$ and $\gamma_{2 b} \simeq 1.6 \times 10^{-2}$ at $T=1400 \mathrm{~K}$ ), while $\alpha$ can be taken as $\alpha \simeq 1 / 6$ with sufficiently good accuracy. Substituting (23) into (19) gives

$C_{\mathrm{H}}=\frac{1}{G H} \frac{k_{2 f} k_{3 f} C_{\mathrm{H}_{2}}^{2}}{k_{1 b} k_{4 f} C_{\mathrm{M}} C_{\mathrm{O}_{2}}}\left(\frac{k_{1 f}}{k_{4 f} C_{\mathrm{M}}} \frac{f+G}{\alpha f+G}-1\right)$,

the desired expression for use in Eq. (11), while from (17) with use of (19) and (27) the O-atom concentration reduces to

$C_{0}=\frac{\alpha f+G}{f+G} \frac{k_{3 f} C_{\mathrm{H}_{2}}}{G k_{1 b}}\left(\frac{k_{1 f}}{k_{4 f} C_{\mathrm{M}}} \frac{f+G}{\alpha f+G}-1\right)$.

It is of interest that, according to (23), (27) and (28), in the steady-state approximation adopted here the concentrations of $\mathrm{OH}$, $\mathrm{H}$ and $\mathrm{O}$, vanish as the temperature approaches the crossover value $T_{c}$, defined by the condition

$k_{1 f}=\frac{\alpha f+G}{f+G} k_{4 f} C_{\mathrm{M}}$,

giving a value that depends on the composition through the functions $f$ and $G$. The concentration of the hydroperoxyl radical, given from (18), (19) and (21) by

$C_{\mathrm{HO}_{2}}=\frac{k_{3 f}}{(f+G) k_{7 f}} C_{\mathrm{H}_{2}}$,

reaches a nonzero value at the crossover temperature and is positive also for $T<T_{c}$. At temperatures below $T_{c}$ the steady-state approximation predicts $C_{O}=C_{\mathrm{OH}}=C_{\mathrm{H}}=0$, so that the reaction rate in Eq. (11) is cut off at that temperature.

The explicit rate expression for the global reaction (12) can be further simplified by noting that, because of the small value of $\gamma_{2 b}$ at temperatures of practical interest, the departures of the factor $H$ from unity in (24) are negligible at the lean equivalence ratios of interest here, and therefore one can use $H=1$ in (27), thereby yielding for the one-step rate

$\omega=\omega_{4 f}=\frac{1}{G}\left(\frac{k_{1 f}}{k_{4 f} C_{\mathrm{M}}} \frac{f+G}{\alpha f+G}-1\right) \frac{k_{2 f} k_{3 f}}{k_{1 b}} C_{\mathrm{H}_{2}}^{2}$ if $k_{1 f}>k_{4 f} C_{\mathrm{M}}(\alpha f+G) /(f+G)$ and $\omega=0$ otherwise, with $G$ and $f$ evaluated from (20) and (21). In the formal one-step result without this approximation, there is an additional factor of $H$ in the denominator of (31). Implications of (31) both with and without this additional factor will be explored.

\section{The lean flammability limit}

In lean premixed flames, the chemical reaction takes place near the hot boundary in a thin layer where the temperature is above its crossover value defined by (29). Since use of this formula involves evaluating $f$, it is evident from (21) that the $\mathrm{H}_{2}$ concentration in the reaction zone plays a role. Because of the presence of the upstream convective-diffusive zone, in this layer the fuel concentration is small compared with its initial value and in the planar reaction zone takes on values of the order

$C_{\mathrm{H}_{2 c}} \sim \frac{T_{\infty}-T_{c}}{T_{\infty}-T_{u}} L_{\mathrm{H}_{2}} C_{\mathrm{H}_{2 u}}$,

where $T_{\infty}$ represents the burnt temperature (the adiabatic flame temperature), and the subscript $u$ denotes conditions in the unburnt mixture. The hydrogen Lewis number $L_{\mathrm{H}_{2}}$ appears in the above expression due to differential diffusion effects in the preheat region [19].

According to the steady-state description (27), H atoms can exist only within this thin layer where $T_{c}<T<T_{\infty}$, with a small concentration that determines the rate of the overall $\mathrm{H}_{2}$-oxidation reaction (12). Clearly, the flame can no longer exist if the temperature remains below crossover throughout, so that the flammability limit corresponds to conditions such that $T_{\infty}=T_{c}$, an equation that can be used in calculating the critical value of the equivalence ratio at the lean flammability limit, $\phi_{l}$, of the planar flame. To determine the value of $T_{c}$ at the flammability limit, $\left(T_{c}\right)_{l}$, it is necessary to observe from (32) that $C_{\mathrm{H}_{2 c}}$ vanishes at $T_{\infty}=T_{c}$, so that the factor $(\alpha f+G) /(f+G)$ in (29) must be taken as unity according to (20) and (21), that is, $f=0$ and $G=1+\gamma_{3 b}$. Equation (29) thus provides the simple expression $k_{1 f}=k_{4 f} C_{\mathrm{M}}$ at the lean flammability limit.

To use this result for finding $\phi_{l}$ and $\left(T_{C}\right)_{l}$, it may be observed that the third-body efficiency factor, appearing in the equation for $C_{\mathrm{M}}$ given below (12), reduces to $\left(15 X_{\mathrm{H}_{2} \mathrm{O}}+1\right)$ with $X_{\mathrm{H}_{2} \mathrm{O}}=$ $2 \phi /(4.76+\phi)$, the burnt gas value, giving a value of $T_{c}$ that depends on the equivalence ratio. Representative results are shown in Fig. 6 for $p=1$ atm and $p=10$ atm. The figure also exhibits the adiabatic flame temperature $T_{\infty}$ obtained from chemical equilibrium for the same values of the pressure. For $p=1 \mathrm{~atm}$, the initial temperature in this figure is taken to be $T_{u}=300 \mathrm{~K}$; for $p=10 \mathrm{~atm}$ the value $T_{u}=580 \mathrm{~K}$ is selected here because this value corresponds to a gas mixture preheated from atmospheric conditions through an isentropic compression, of interest in engine applications. The figure illustrates the slight increase of $\left(T_{C}\right)_{l}$ with $\phi$, associated with the increase of $X_{\mathrm{H}_{2} \mathrm{O}}$, and the well-known stronger increase of $T_{\infty}$ with $\phi$. For a given pressure, the crossing point between the two curves in Fig. 6 determines the critical values of the equivalence ratio and crossover temperature at the lean flammability limit of the steady planar flame, yielding $\phi_{l}=0.251$ and $\left(T_{c}\right)_{l}=1080 \mathrm{~K}$ for $T_{u}=300 \mathrm{~K}$ and $p=1 \mathrm{~atm}$ and $\phi_{l}=0.279$ and $\left(T_{c}\right)_{l}=1380 \mathrm{~K}$ for $T_{u}=580 \mathrm{~K}$ and $p=10 \mathrm{~atm}$.

From the crossing points in Fig. 6, flammability limits were calculated as functions of pressure for four different initial temperatures. The results are shown by the solid curves in Fig. 7. Also shown (by dashed curves) in the figure are the calculated flame temperatures at the limit for the two extreme cases. The results illustrate the increase of $\phi_{l}$ and $\left(T_{c}\right)_{l}$ with $p$, arising from the associated increase in $C_{\mathrm{M}}$, the three-body recombination becoming 


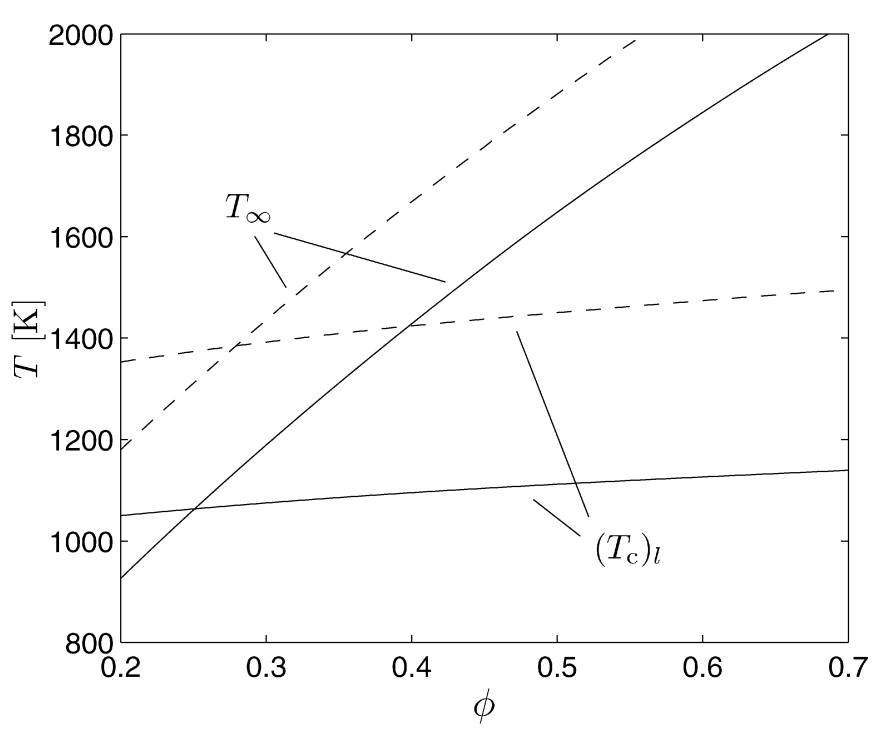

Fig. 6. The variation with equivalence ratio of the $\mathrm{H}_{2}$-air adiabatic flame temperature $T_{\infty}$, as obtained from chemical equilibrium, and of the crossover temperature at the lean flammability limit $\left(T_{c}\right)_{l}$, as obtained for atmospheric pressure from $k_{1 f}=k_{4 f} C_{\mathrm{M}}$, for $p=1 \mathrm{~atm}$ and $T_{u}=300 \mathrm{~K}$ (solid line) and for $p=10 \mathrm{~atm}$ and $T_{u}=580 \mathrm{~K}$ (dashed line).

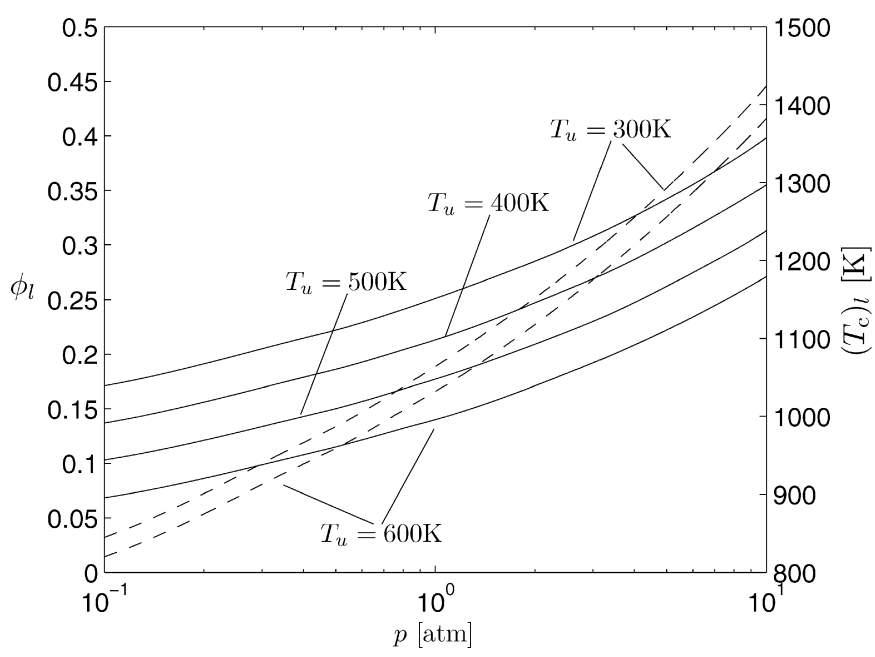

Fig. 7. The calculated variation of the equivalence ratio $\phi_{l}$ (solid curves) and flame temperature $\left(T_{C}\right)_{l}$ (dashed curves) with pressure at the lean flammability limit for four different values of the initial temperature $T_{u}$.

relatively faster than the two-body branching with increasing pressure; the strength of this dependence is seen to increase with $p$. In these calculations, falloff was included for $k_{4 f}$ as described previously, and $T_{\infty}$ was obtained from a chemical-equilibrium routine [20]. The temperatures obtained are within a few degrees of those found in the final downstream conditions predicted by COSILAB [10] with detailed chemistry and within $10 \mathrm{~K}$ to $20 \mathrm{~K}$ of those calculated for complete combustion to $\mathrm{H}_{2} \mathrm{O}$ at these relatively lowtemperature, near-limit conditions, the largest differences occurring at the largest values of $\phi$ in the figure. The results shown in Fig. 7 thus are accurate within a few percent, comparable to the accuracy of the plotting. They do, however, ignore influences of heat losses on flammability limits, which would tend to increase $\phi_{l}$, and they exclude reactions that may occur below crossover (increasingly important with increasing pressure) and effects of nonplanar differential diffusion, both of which tend to decrease $\phi_{l}$, the latter significantly.
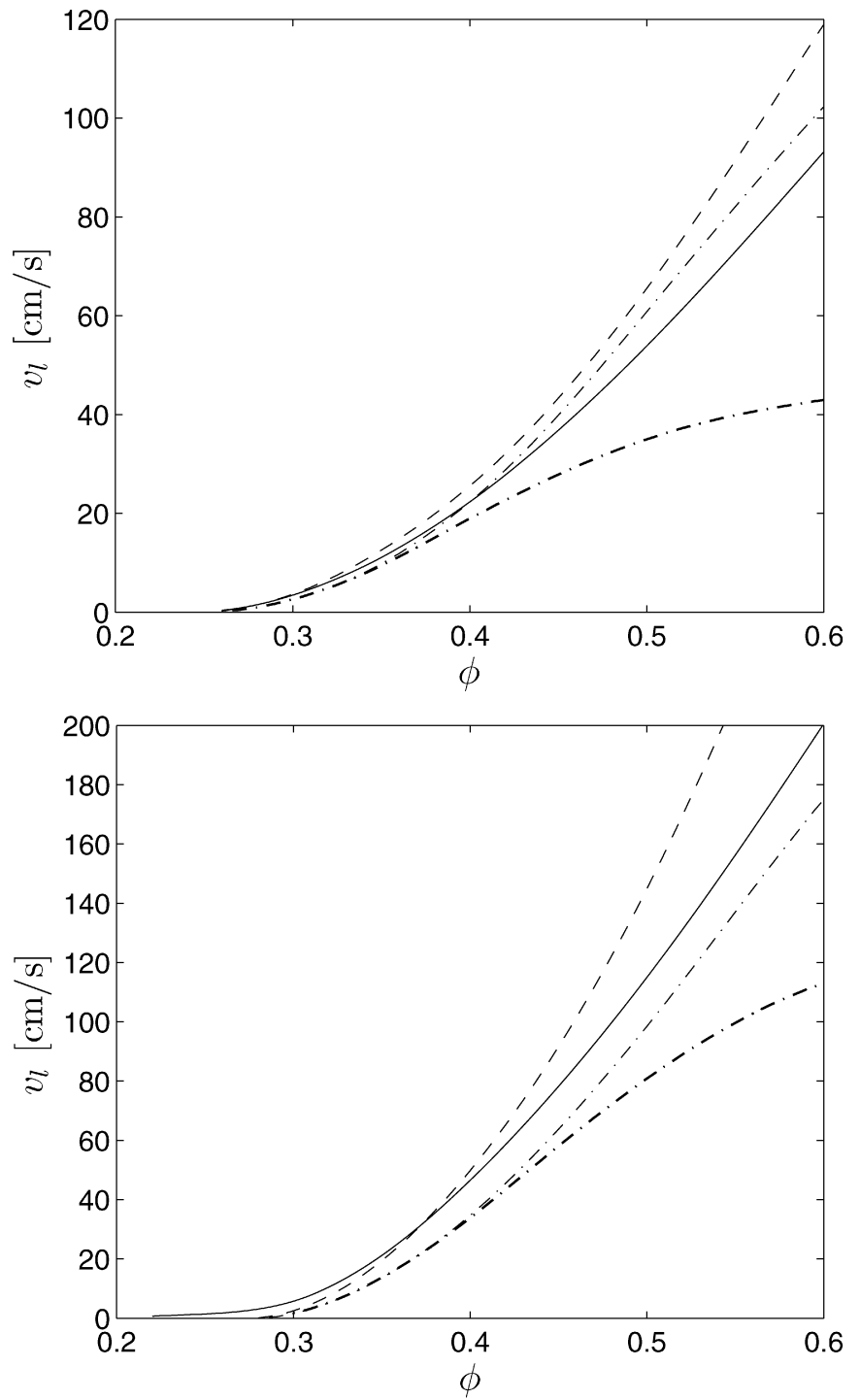

Fig. 8. The variation with equivalence ratio of the propagation velocity of a premixed hydrogen-air flame for $p=1$ atm and $T_{u}=300 \mathrm{~K}$ (upper plot) and for $p=10 \mathrm{~atm} T_{u}=580 \mathrm{~K}$ (lower plot) as obtained from numerical integrations with detailed chemistry (solid curve), with the 7-step mechanism of Table 1 (dashed curve), with the one-step reduced mechanism for $H=1$ (thin dot-dashed curve), and with the one-step reduced mechanism for variable $H$ (thick dot-dashed curve).

\section{The flame propagation velocity}

The one-step kinetics in (31) was employed in computations of adiabatic flame propagation velocities for the conditions of pressure and initial temperature of Fig. 6, giving results that are compared in Fig. 8 with results of computations for detailed and 7-step chemistry. The computations are based on the conservation equations for fuel and energy which, in the thin reactive-diffusive layer, reduce to

$\rho \frac{D_{T}}{L_{\mathrm{H}_{2}}} \frac{\mathrm{d}^{2} Y_{\mathrm{H}_{2}}}{\mathrm{~d} n^{2}}=2 W_{\mathrm{H}_{2}} \omega$

and

$\rho c_{p} D_{T} \frac{\mathrm{d}^{2} T}{\mathrm{~d} n^{2}}=-2 W_{\mathrm{H}_{2}} q \omega$

if $n$ is defined as the coordinate normal to the reaction layer, with $n=0$ at crossover, and $Y_{\mathrm{H}_{2}}$ and $W_{\mathrm{H}_{2}}$ are the mass fraction and molecular weight of $\mathrm{H}_{2}$; here $q=-h_{\mathrm{H}_{2} \mathrm{O}}^{0} / W_{\mathrm{H}_{2}}$ is the amount of 
heat release per unit mass of fuel consumed, with $h_{\mathrm{H}_{2} \mathrm{O}}^{0}$ representing the enthalpy of formation per mol of water vapor. Since the reaction layer is relatively thin, the density, $\rho$, thermal diffusivity, $D_{T}$, and specific heat at constant pressure, $c_{p}$, can be taken as constants evaluated at the burnt temperature $T_{\infty}$ and with the equilibrium composition given below in (38). The approximate expression $\rho D_{T}=2.58 \times 10^{-5}(T / 298)^{0.7} \mathrm{~kg} /(\mathrm{ms})$ [21] is used in evaluating the thermal diffusivity; although this approximation applies to methane-air flames, since the thermal conductivity and heat capacity of lean hydrogen flames are dominated by the values for nitrogen and oxygen, the result also is sufficiently accurate here, as tests using more complex NASA polynomials verified. For hydrogen, $L_{\mathrm{H}_{2}}=0.3$.

Integrating twice a linear combination of the above two equations with boundary conditions $Y_{\mathrm{H}_{2}}=T-T_{\infty}=0$ as $n \rightarrow \infty$ yields

$Y_{\mathrm{H}_{2}}=L_{\mathrm{H}_{2}} \frac{T_{\infty}-T}{q / c_{p}}$,

which can be evaluated at $n=0$ to determine the value of fuel mass fraction at the crossover temperature $T=T_{c}$, namely

$Y_{\mathrm{H}_{2 c}}=L_{\mathrm{H}_{2}} \frac{T_{\infty}-T_{c}}{q / c_{p}}$.

Integrating (33) once after multiplication by $\mathrm{d} Y_{\mathrm{H}_{2}} / \mathrm{d} n$ yields

$$
\begin{aligned}
v_{l} & =-\left(\frac{\rho D_{T}}{\rho_{u} Y_{\mathrm{H}_{2 u}} L_{\mathrm{H}_{2}}} \frac{\mathrm{d} Y_{\mathrm{H}_{2}}}{\mathrm{~d} n}\right)_{c} \\
& =\frac{2}{\rho_{u} Y_{\mathrm{H}_{2 u}}}\left(\rho \frac{D_{T}}{L_{\mathrm{H}_{2}}} W_{\mathrm{H}_{2}} \int_{0}^{Y_{\mathrm{H}_{2 c}}} \omega \mathrm{d} Y_{\mathrm{H}_{2}}\right)^{1 / 2},
\end{aligned}
$$

for the burning velocity after application of the matching condition obtained from the solution for $Y_{\mathrm{H}_{2}}$ in the preheat zone [19].

In evaluating the integral of $\omega$ in (37) it is necessary to account for changes in the fuel concentration, which appears as a quadratic factor in (31), and it is also necessary to take into account the variation of the associated temperature decrement $T_{\infty}-T$, to be determined from (35), which is important because of the temperature sensitivity of the reaction-rate constants, especially that of $1 \mathrm{f}$. The result depends also on the oxygen and water-vapor mol fractions because they appear in (21) and (22); they can be evaluated from their equilibrium values

$X_{\mathrm{H}_{2} \mathrm{O}} /(2 \phi)=X_{\mathrm{O}_{2}} /(1-\phi)=1 /(4.76+\phi)$,

which remain constant in the reaction zone at leading order. Because of the complexity of the expression for $\omega$, the integral in (37) is evaluated numerically, but computationally in principle this is relatively simple compared to numerical integrations of the differential equations, which were performed with COSILAB [10] for generating the results for the 7-step and detailed chemistry.

For atmospheric pressure, the agreement between the detailed and short mechanisms seen in Fig. 8 is excellent, with values of $v_{l}$ that differ by less than $20 \%$ for $\phi_{l}<\phi<0.6$. The steady-state description predicts accurately the lean flammability limit $\left(v_{l}=0\right.$ according to the approximations that lead to the one-step description), which also agrees well with the detailed-chemistry prediction. The burning velocity obtained from the steady-state approximations also agrees well with the detailed-chemistry results, until about $\phi=0.4$, at which point whether the factor $H$ is included in (31) begins to make a great difference, the prediction of the strictly correct imposition of steady states yielding burning velocities that are much too low at higher $\phi$, while the simpler formula with the near-limit value $H=1$ produces burning velocities in rather good agreement with detailed-chemistry predictions. Since the plots in Fig. 5 indicate that the steady-state approximation becomes poor at $\phi=0.5$, the disagreement is understandable, the true $\mathrm{H}$-atom concentration significantly exceeding its steady-state value at the higher equivalence ratios. The one-step result for $H=1$ is seen in the top plot of Fig. 8 fortuitously to agree even better than the 7 step results with the predictions of the detailed chemistry, except very near the flammability limit. For $p=1 \mathrm{~atm}$, the departures in $v_{l}$ of the one-step description with $H=1$ from the detailedchemistry results remain below $15 \%$ for $\phi_{l}<\phi<0.6$.

From the bottom plot in Fig. 8 it is seen that at 10 atm the departures of the predictions of the one-step mechanism from those of the 7-step mechanism on which it is based are greater than at $1 \mathrm{~atm}$. In general, decreasing pressure improves the burningvelocity agreement of the one-step and 7-step mechanisms (and also improves the agreement of the 7-step mechanism with detailed chemistry), and at subatmospheric pressures the one-step mechanism is quite good for lean flames. The differences between the one-step and 7-step mechanisms is greater at $10 \mathrm{~atm}$ because the approximation employed for the diffusivity in the one-step calculations is in poorer agreement with the COSILAB transport data at this higher pressure and temperature; the steady-state approximations for the intermediates are as good or better at the higher pressure, so that the one-step reaction-rate expression is justified just as well. It is seen that this difference for $10 \mathrm{~atm}$ is now so great that the burning velocity predicted by the one-step mechanism lies below that of the detailed mechanism over the entire range of lean equivalence ratios, irrespective of whether the further approximation $H=1$ is introduced. This approximation, however, continues to describe the overall reaction rate better than full, correct imposition, of all steady states. The predictions of the onestep and 7-step mechanisms are very close near the flammability limit of the one-step mechanism, but in this range at 10 atm they both significantly underpredict the burning velocity of the detailed mechanism.

This last difference is due to the approach to the third explosion limit with detailed chemistry; the formation of $\mathrm{H}_{2} \mathrm{O}_{2}$ from $\mathrm{HO}_{2}$ and its regeneration of active radicals is not entirely negligible at $10 \mathrm{~atm}$. This is seen in the lower plot of Fig. 8 to have a potentially large effect on the lean flammability limit, if it is defined by $v_{l} \approx 0$. To that extent, the flammability limits predicted in the preceding section are inaccurate at high pressure. Since heat losses, however, typically extinguish flames readily if their burning velocities are below about $5 \mathrm{~cm} / \mathrm{s}$, the limits predicted in the preceding section may remain reasonable for planar flames up to $10 \mathrm{~atm}$. In general, the detailed mechanism predicts positive burning velocities for all equivalence ratios, but at very low equivalence ratios these velocities are extremely small, although they increase significantly with increasing $p$ at any given $T_{u}$. The 7-step mechanism is seen to provide good burning-velocity agreement with the detailed mechanism at $10 \mathrm{~atm}$ with $T_{u}=580 \mathrm{~K}$ for $0.33<\phi<0.43$.

With these comparisons in mind, it is of interest to exhibit the burning-velocity predictions of the one-step mechanism with $H=1$ for various pressures and initial temperatures, for $0.1<\phi<$ 0.6 . Fig. 9 shows such predictions, demonstrating how $v_{l}$ increases with $T_{u}$ and varies much less strongly with $p$. The results in Fig. 9 are best at low pressure, the accuracy being degraded at elevated pressure, as explained above.

\section{Arrhenius approximation}

It is of interest to test how well the present results can be matched by one-step Arrhenius reaction-rate approximations. Such approximations have been investigated previously on the basis of experimental [22] and numerical [5] results. Although the burning velocities of Fig. 9 could be used for these tests, it is in a sense more fundamental to work with the rate expression of Eq. (31), employing the flame-structure solutions to construct an Arrhe- 


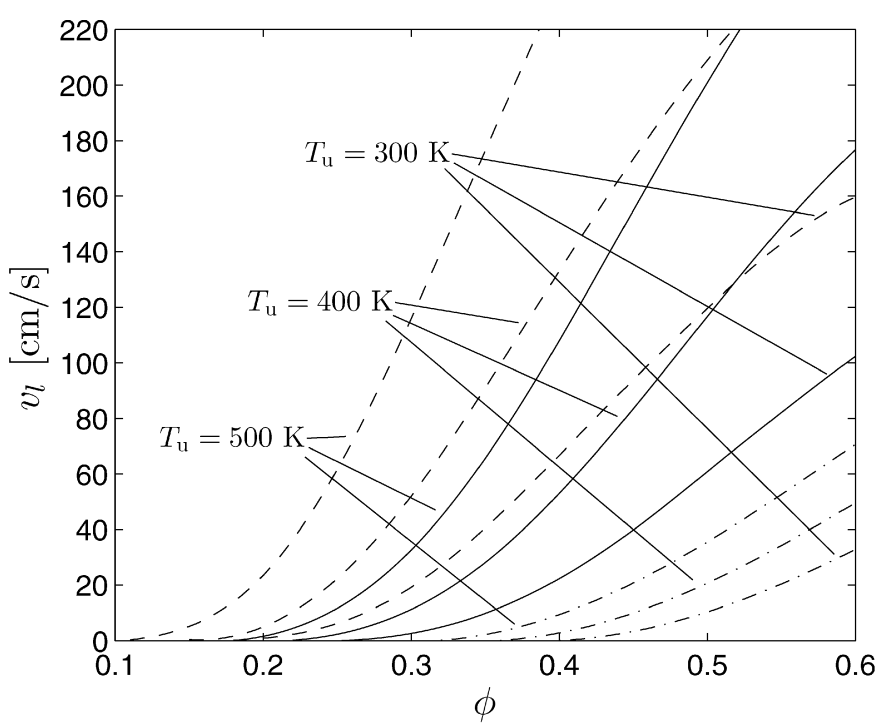

Fig. 9. The variation with equivalence ratio of the propagation velocity of planar premixed hydrogen-air flames predicted by the one-step mechanism with $H=1$ for $p=0.1$ atm (dashed curves), $p=1$ atm (solid curves) and $p=10$ atm (dotdashed curves) for three different initial temperatures.

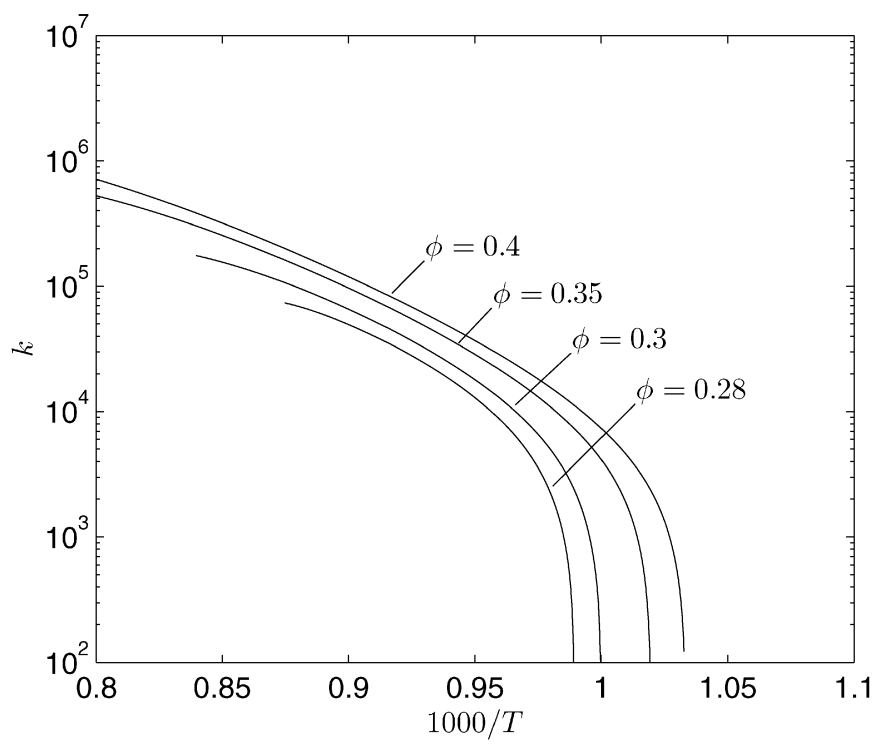

Fig. 10. The variation with temperature of the factor $k=\left(k_{2 f} k_{3 f}\right) /\left(G k_{1 b}\right)\left\{k_{1 f}(f+\right.$ $\left.G) /\left[k_{4 f} C_{\mathrm{M}}(\alpha f+G)\right]-1\right\}$ for four different equivalence ratios at $p=1 \mathrm{~atm}$ and $T_{u}=300 \mathrm{~K}$.

nius plot of the quantity multiplying $C_{\mathrm{H}_{2}}^{2}$ in order to obtain a second-order rate expression of the form $\omega=B \exp \left(-T_{a} / T\right) C_{\mathrm{H}_{2}}^{2}$. Fig. 10 shows such plots for four different equivalent ratios at $p=1 \mathrm{~atm}$ and $T_{u}=300 \mathrm{~K}$, with $k$ denoting in the figure the quantity multiplying $\mathrm{C}_{\mathrm{H}_{2}}^{2}$ on the right-hand side of (31). It is seen from this figure that, although such an approximation can be fit to the numerical results without excessive inaccuracy in an intermediate temperature range, the resulting activation temperatures vary appreciably, especially as crossover is approached. Away from crossover, the resulting values of the overall activation temperatures are of the order of $T_{a} \simeq 20000 \mathrm{~K}$, somewhat larger than the values reported earlier [5,22], which correspond however to different conditions. In view of the plot, it is clear that a simple Arrhenius expression does not suffice to reproduce correctly the profile of the overall reaction rate near the lean flammability limit and that future efforts to derive a simplified reaction rate must account for the effect of the crossover temperature.

\section{Limitations of the one-step chemistry}

Further study of the limitations of the one-step chemistry is desirable. The explicit steady-state expression (27) is tested in Fig. 2, which includes comparisons of the $\mathrm{H}$-atom profiles determined numerically on the basis of the 7-step mechanism with those determined from evaluating (27). In the evaluations, use has been made of the profiles of reactant and water-vapor mol fractions and of temperature obtained numerically with the 7-step mechanism. It can be seen that the accuracy of the steady-state expression is best at very lean conditions, but it worsens as the mixture becomes richer, in agreement with the observations of Fig. 5. For the three conditions plotted in Fig. 2, it is evident that the steadystate assumption clearly fails at crossover, where the steady state predicts $\mathrm{H}$ atoms to disappear abruptly, thereby giving a profile with a discontinuous slope. Diffusive transport enters to remove this discontinuity, so that a smooth corner-layer profile replaces the abrupt change of the steady-state predictions when the 7-step mechanism is employed in the computations. In addition, it is seen in Fig. 2 that for all three conditions shown the steady-state approximation tends to overpredict the radical peak, giving values that exceed those obtained with detailed kinetics by roughly $50 \%$ for $\phi=0.35$. Analysis of the corner layer, in which the steady-state approximations fail, will provide corrections to burning velocities (37) predicted by the one-step mechanism.

Besides this inaccuracy at small $\phi$, the one-step chemistry fails if $\phi-\phi_{l}$ becomes too large. In deriving the first equality in (11) from (8) we have assumed that in the reaction layer radicals exhibit concentrations that are much smaller than $\mathrm{H}_{2}$ concentrations, a condition that can be seen to be clearly satisfied by the radicals plotted for $\phi=0.3$ in Fig. 3 but not so clearly by those corresponding to $\phi=0.5$ shown in Fig. 4. Radical concentrations, which are very small for flames near the flammability limit, become increasingly larger for increasing values of the equivalence ratio, causing the one-step description to break down. If $\mathrm{H}$ is considered to be the dominant radical in the radical pool, which can be seen to apply increasingly as the mixture becomes richer, the validity of the reduced kinetics is associated with the condition that $C_{\mathrm{H}} \ll \mathrm{C}_{\mathrm{H}_{2}}$ in the reaction layer. To determine the characteristic value of $C_{\mathrm{H}}$ in the reaction zone, use may be made of (27), taking $H=1$ for simplicity, with

$\left(\frac{k_{1 f}}{k_{4 f} C_{\mathrm{M}}} \frac{f+G}{\alpha f+G}-1\right) \sim \frac{T_{a_{1 f}}}{T_{c}} \frac{T_{\infty}-T_{c}}{T_{c}}$,

implied by an expansion for $T_{\infty}$ near $T_{c}$. The result is

$C_{\mathrm{H}_{c}}=\frac{1}{G} \frac{k_{2 f} k_{3 f} C_{\mathrm{H}_{2}}^{2}}{k_{1 b} k_{4 f} C_{\mathrm{M}} C_{\mathrm{O}_{2}}} \frac{T_{a_{1 f}}}{T_{c}} \frac{T_{\infty}-T_{c}}{T_{c}}$.

Furthermore, in the first approximation one may employ (32) to estimate the amount of $\mathrm{H}_{2}$ in the reaction layer and take $\mathrm{C}_{\mathrm{O}_{2}} \sim$ $C_{\mathrm{O}_{2 u}}(1-\phi)$. With these simplifications, the condition that $C_{\mathrm{H}} \ll$ $\mathrm{C}_{\mathrm{H}_{2}}$ in the reaction layer reduces to

$\left(\frac{2 \phi \beta L_{\mathrm{H}_{2}} k_{2 f} k_{3 f}}{(1-\phi) G k_{1 b} k_{4 f} C_{\mathrm{M}}}\right)\left(\frac{T_{\infty}-T_{c}}{T_{\infty}-T_{u}}\right)^{2} \ll 1$,

where $\beta=T_{a_{1 f}}\left(T_{\infty}-T_{u}\right) / T_{c}^{2}$ is the relevant Zeldovich number.

The restriction given by (41) can be used to estimate the validity of the proposed one-step reduced kinetics for given conditions of pressure, composition and initial temperature. In the computation, the plots of Fig. 6 may be used to obtain $T_{\infty}$ and $T_{c}=\left(T_{C}\right)_{l}$, and $G$ may be taken equal to unity. Evaluating the left-hand side of (41) with the equilibrium mol fractions given in (38) to compute 
the third-body efficiency of reaction $4 \mathrm{f}$ and with the reaction-rate constants evaluated at $T_{c}$ yields values of the left-hand side of (41) of $1.8 \times 10^{-2}$ and 0.61 for $\phi=0.3$ and $\phi=0.5$, respectively. The approximate criterion (41) for the validity of the steady-state assumption thus clearly holds for $\phi=0.3$ but is not so clearly satisfied for $\phi=0.5$, a result that might be anticipated from Fig. 5 and that is reflected in Fig. 8. For lean flames with $\phi \gtrsim 0.5$, a twostep description is needed [7].

\section{Conclusions}

This research has derived systematically an explicit one-step reaction-rate expression for the $\mathrm{H}_{2}$ oxidation reaction (12) that provides reasonable accuracy for calculating the lean flammability limit and laminar burning velocities of hydrogen-air systems from the lean limit to equivalence ratios that depend on the pressure and on the initial temperature, but that always are fuel-lean. The explicit reaction-rate formula does not conform to CHEMKIN or COSILAB formulas, for example, and so would require additional programming to be used with those codes, but it is especially well suited for use in future time-dependent, multidimensional codes for addressing hydrogen-air laminar or turbulent (DNS) flame propagation in complex geometries, where descriptions employing detailed chemistry would be too large to be handled by existing or near-future computers. In the process of deriving the one-step formula, short-chemistry descriptions of nine, eight and seven elementary steps (with rate expressions in formats that do conform to existing codes) were identified and demonstrated to succeed in achieving additional objectives, such as improving predictions of concentration profiles of radicals other than the $\mathrm{H}$ atom (which is predicted well by the one-step mechanism) or extending accurate burning-velocity predictions through stoichiometry to include all fuel-rich systems as well. These short mechanisms could facilitate computations having broader objectives and abilities to handle mechanisms larger than just a few steps.

The one-step mechanism is based on the demonstrated applicability of chemical-kinetic steady-state approximations for all reaction intermediaries, including the $\mathrm{H}$ atom, which is not in steady state in previously derived reduced-chemistry descriptions such as the two-step mechanism that earlier investigations have found to be useful for many purposes. The one-step mechanism applies for final flame temperatures between the crossover temperature (at which the rate of the $\mathrm{H}+\mathrm{O}_{2} \rightarrow \mathrm{OH}+\mathrm{O}$ branching step equals the rate of the $\mathrm{H}+\mathrm{O}_{2}+\mathrm{M} \rightarrow \mathrm{HO}_{2}+\mathrm{M}$ three-body step that leads to recombination) and a higher temperature at which the radical concentrations are too large for an H-atom steady state to be sufficiently accurate. This range of applicability decreases with increasing pressure and vanishes at sufficiently high pressures, approaching the third explosion limit at which $\mathrm{H}_{2} \mathrm{O}_{2}$ becomes an important intermediate radical generator, above about 10 atm for representative normal initial environmental temperatures. At $1 \mathrm{~atm}$ and an initial temperature of $300 \mathrm{~K}$, for example, the one-step mechanism yields the burning velocity with an error less than $15 \%$ all the way from the lean limit of the planar flame, at an equivalence ratio of about 0.25 , to an equivalence ratio above 0.60 if an approximation $(H=1)$ of a small relative rate of the backward step 2, $\mathrm{H}+\mathrm{OH} \rightarrow \mathrm{H}_{2}+\mathrm{O}$, that is, in (24) and (25), $\gamma_{2 b} \rightarrow 0$, is imposed. This accuracy at equivalence ratios above 0.4 , however, is fortuitous since the $\mathrm{H}$-atom steady-state approximation begins to fail badly there. The one-step mechanism can also be applied for instance for the description of cryogenic $\mathrm{H}_{2}-\mathrm{O}_{2}$ deflagrations near the lean flammability limit, of interest in cryogenic rocket engines [5].

Besides being useful in computational studies, the one-step mechanism can facilitate future analytical work. Investigations of the stability of planar flames and of the structure of nonplanar flames near the lean limit can make good use of the one-step re- sults. Lean hydrogen-air deflagrations are known to have diffusivethermal instabilities that lead to cellular flames, and the one-step chemistry derived here can greatly facilitate analyses of cellular structures. Such analyses in the past have generally been based on one-step activation-energy asymptotics, an approach that is merely phenomenological and is not based directly on the underlying chemistry that actually is occurring. The present results now enable these analyses to be revised and tied to the real chemistry. Lean-hydrogen cellular-flame computational works also can make use of the present results numerically.

Further improvement of the chemical-kinetic descriptions developed here would be worthwhile. For example, at the cold end of the reaction layer, very near crossover, a corner layer has been identified here, in which the steady-state approximations that underlie the one-step description fail. Analysis of this very thin corner layer is justified, for example, for generating corrections to the burning velocities predicted from the one-step approximation, leading to improved accuracy. The manner in which steadystate accuracy is lost at higher equivalence ratios also merits further investigations. Preliminary study indicates that the transition from the present one-step regime to previously analyzed two-step regimes is not simple, and the associated chemical-kinetic complexities need further study, not only for improving understanding but also for deriving more accurate burning-rate and speciesprofile results, as well as chemical-kinetic descriptions, that can be used in future investigations of hydrogen-air deflagration structure, propagation and dynamics.

\section{Acknowledgments}

This collaborative research was supported by the Spanish MEC under Projects \# ENE2005-08580-C02-01 and ENE2005-09190C04-01, by the Spanish MICINN under Project \# ENE2008-0615C04, and by the Comunidad de Madrid under Project \# S505/ENE/0229. The work of D.F.G. was supported by the Spanish MICINN through the FPU Program (AP2005-0446).

\section{Appendix A. The steady-state expressions for the 8-step mechanism}

As indicated in the main text, the description of the radicals 0 and $\mathrm{OH}$ given by the 7-step mechanism loses accuracy in flames close to the lean flammability limit. To correct this deficiency, it is necessary to include the shuffle reaction

$\mathrm{H}_{2} \mathrm{O}+\mathrm{O} \stackrel{8}{\rightleftharpoons} \mathrm{OH}+\mathrm{OH}$

in the short mechanism. When this is done, the resulting profiles of $\mathrm{O}$ and $\mathrm{OH}$ agree well with those calculated on the basis of the detailed chemistry, as can be seen in Fig. A.1. In particular, the agreement of the $\mathrm{O}$ and $\mathrm{OH}$ profiles is much better than that seen in Fig. 3 for the 7-step mechanism; the agreement of the $\mathrm{H}$ profile is so good that the solid and dashed curves cannot be distinguished. As mentioned before, the addition of reaction 8 does not affect significantly the $\mathrm{H}$-atom profile, which remains practically unperturbed from that obtained with the 7-step mechanism, so that reaction 8 can be discarded for simplicity in computing the global rate of the one-step reduced kinetics, as is done in the main text. If, however, there is interest in the $\mathrm{O}$ and $\mathrm{OH}$ profiles under these conditions, then the further considerations given in this appendix become useful.

Inclusion of (A.1) in the mechanism modifies the steady-state expressions for the radicals. The starting equations take the form

$0=\omega_{1}-\omega_{2}-\omega_{8}$,
$0=\omega_{1}+\omega_{2}-\omega_{3}+2 \omega_{5 f}-\omega_{7 f}+2 \omega_{8}$, 


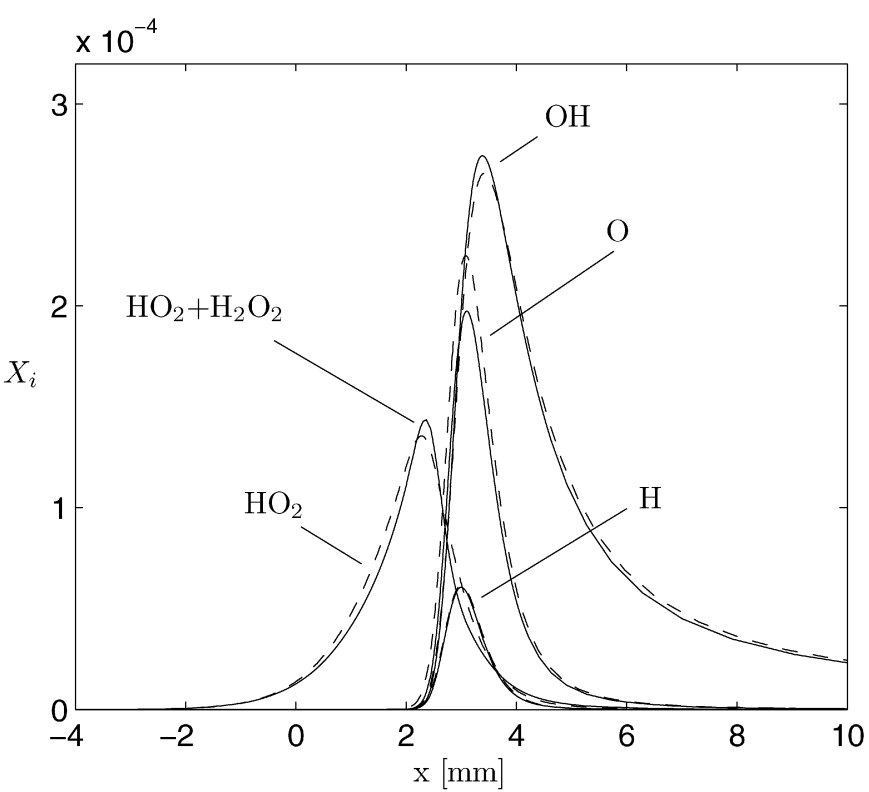

Fig. A.1. Profiles of radical mole fractions in the flame, as obtained from detailed kinetics (solid curves) and from the 8-step mechanism (dashed curves) for $\phi=0.3$, $p=1 \mathrm{~atm}$ and $T_{u}=300 \mathrm{~K}$; the sum of the $\mathrm{HO}_{2}$ and $\mathrm{H}_{2} \mathrm{O}_{2}$ mole fractions is shown for the detailed chemistry because $\mathrm{H}_{2} \mathrm{O}_{2}$ formation is absent in the short mechanism.

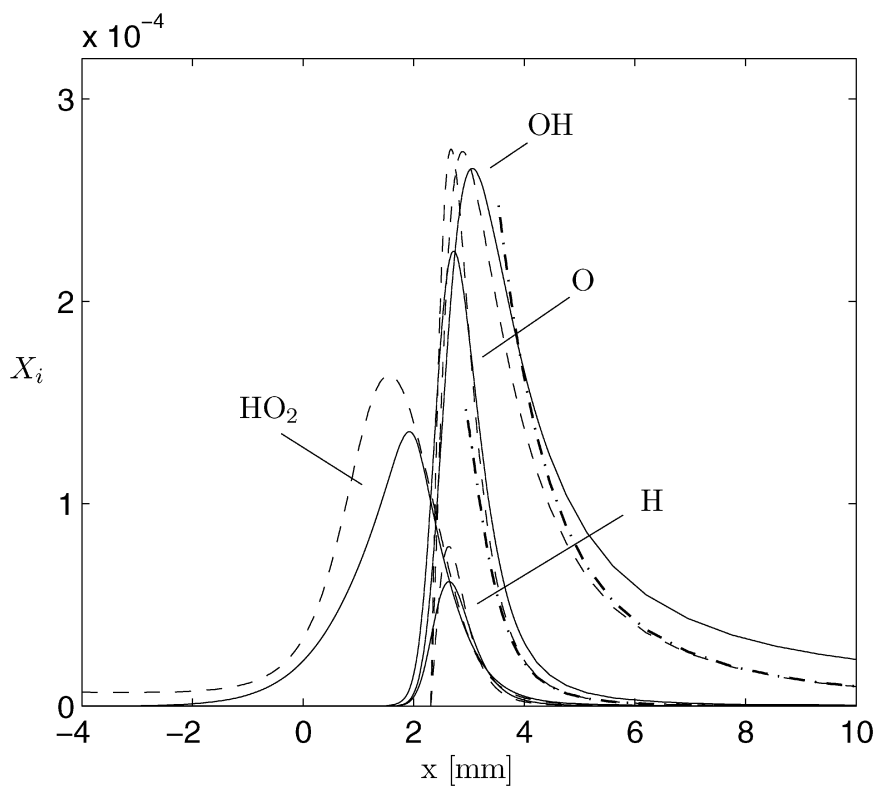

Fig. A.2. Profiles of radical mole fractions in the flame as obtained with the 8step mechanism (the 7-step mechanism augmented with the shuffle reaction 8) (solid curves), from numerical evaluations of the steady-state expressions (A.6)(A.9) (dashed curves) and from use of the formulas (A.15) and (A.16) (dot-dashed curves), for $\phi=0.3, p=1 \mathrm{~atm}$ and $T_{u}=300 \mathrm{~K}$.

$0=-\omega_{1}+\omega_{2}+\omega_{3}-\omega_{4 f}-\omega_{5 f}-\omega_{6 f}$,

and

$0=\omega_{4 f}-\omega_{5 f}-\omega_{6 f}-\omega_{7 f}$.

Appropriate manipulation then leads to the exact expressions

$C_{0}=\frac{\alpha f+\tilde{G}}{f+\tilde{G}} \frac{k_{3 f} C_{\mathrm{H}_{2}}}{\tilde{G} k_{1 b}}\left(\frac{k_{1 f}}{k_{4 f} C_{\mathrm{M}}} \frac{f+\tilde{G}}{\alpha f+\tilde{G}}-1\right)$,

$C_{\mathrm{OH}}=\frac{1}{\tilde{H}} \frac{\left(k_{2 f} C_{\mathrm{H}_{2}}+k_{8 f} C_{\mathrm{H}_{2} \mathrm{O}}\right)}{k_{1 b}}\left(\frac{k_{1 f}}{k_{4 f} C_{\mathrm{M}}} \frac{f+\tilde{G}}{\alpha f+\tilde{G}}-1\right)$

$$
\begin{aligned}
& C_{\mathrm{H}}=\frac{1}{\tilde{G} \tilde{H}} \frac{\left(k_{2 f} C_{\mathrm{H}_{2}}+k_{8 f} C_{\mathrm{H}_{2} \mathrm{O}}\right) k_{3 f} C_{\mathrm{H}_{2}}}{k_{1 b} k_{4 f} C_{\mathrm{M}} C_{\mathrm{O}_{2}}}\left(\frac{k_{1 f}}{k_{4 f} C_{\mathrm{M}}} \frac{f+\tilde{G}}{\alpha f+\tilde{G}}-1\right), \\
& C_{\mathrm{HO}_{2}}=\frac{k_{3 f}}{(f+\tilde{G}) k_{7 f}} C_{\mathrm{H}_{2}},
\end{aligned}
$$

where the functions $\tilde{G}$ and $\tilde{H}$ are determined from the solution of the coupled equations

$$
\begin{aligned}
\tilde{H}= & \frac{1}{2}+\frac{1}{2}\left[1+4\left(\frac{\gamma_{2 b} f}{1-\gamma_{8 f}}+\frac{\gamma_{8 b} \tilde{G}}{f}\right) \frac{f+\tilde{G}}{\alpha f+\tilde{G}}\right. \\
& \left.\times\left(\frac{k_{1 f}}{k_{4 f} C_{\mathrm{M}}} \frac{f+\tilde{G}}{\alpha f+\tilde{G}}-1\right)\right]^{1 / 2}
\end{aligned}
$$

and

$$
\begin{aligned}
\tilde{G} & -\gamma_{3 b}-\frac{\alpha f+\tilde{G}}{f+\tilde{G}} \gamma_{8 f} \tilde{H}+\frac{\gamma_{8 b} \tilde{G}}{f \tilde{H}}\left(\frac{k_{1 f}}{k_{4 f} C_{\mathrm{M}}} \frac{f+\tilde{G}}{\alpha f+\tilde{G}}-1\right) \\
& -\frac{2 f+\tilde{G}}{f+\tilde{G}}=0,
\end{aligned}
$$

with

$\gamma_{8 f}=\frac{k_{8 f} C_{\mathrm{H}_{2} \mathrm{O}}}{k_{8 f} C_{\mathrm{H}_{2} \mathrm{O}}+k_{2 f} C_{\mathrm{H}_{2}}}$

and

$\gamma_{8 b}=\frac{k_{5 f}+k_{6 f}}{k_{7 f}} \frac{k_{8 b}\left(k_{8 f} C_{\mathrm{H}_{2} \mathrm{O}}+k_{2 f} C_{\mathrm{H}_{2}}\right)}{k_{1 b} k_{4 f} C_{\mathrm{M}} C_{\mathrm{O}_{2}}}$.

It is easy to see that when $k_{8 f}=k_{8 b}=0$ the solution reduces to $\tilde{H}=H$ and $\tilde{G}=G$, and the steady-state expressions of the 7-step mechanism given in (23), (27), (28) and (30) are recovered. By comparing the solid and dashed curves in Fig. A.2, where the temperature and concentrations of the main species are obtained from the 8-step mechanism, it is seen that the steady-state approximation is reasonably good for all four radicals under these conditions. Note that the $\mathrm{O}, \mathrm{OH}$ and $\mathrm{H}$ concentrations given by (A.6)-(A.8) vanish at a crossover temperature defined by the equation

$k_{1 f}=\frac{\alpha f+\tilde{G}}{f+\tilde{G}} k_{4 f} C_{\mathrm{M}}$,

which differs from the expression (29) of the 7-step approximation, although their limiting forms at very lean conditions $k_{1 f}=$ $k_{4 f} C_{\mathrm{M}}$ are identical, indicating that inclusion of reaction 8 does not modify the lean flammability results given in Fig. 7 .

A disadvantage of Eqs. (A.6)-(A.9) is the necessity of solving complex algebraic equations numerically. Explicit expressions can be derived in the limit $C_{\mathrm{H}_{2}} \ll 1$ of small hydrogen concentrations, when the radicals concentrations achieve small values $C_{\mathrm{O}} \propto \mathrm{C}_{\mathrm{H}_{2}}$, $C_{\mathrm{OH}} \propto C_{\mathrm{H}_{2}}^{1 / 2}, C_{\mathrm{H}} \propto C_{\mathrm{H}_{2}}^{3 / 2}$, and $C_{\mathrm{HO}_{2}} \propto C_{\mathrm{H}_{2}}$. Under those conditions, reaction 8 becomes faster than the others, and can be assumed to be in partial equilibrium, while reactions $2,5 \mathrm{f}$ and $6 \mathrm{f}$ become negligibly slow, and can be correspondingly discarded in the steadystate equations (A.2)-(A.5). The problem reduces to that of solving the partial-equilibrium equation $\omega_{8}=0$, together with the $\omega_{8}$-free linear combination of (A.2) and (A.3), $3 \omega_{1}-\omega_{3}-\omega_{7 f}=0$, and with the simplified forms, $-\omega_{1}+\omega_{3}-\omega_{4 f}=0$ and $\omega_{4 f}-\omega_{7 f}=0$, of (A.4) and (A.5). The solution provides

$$
\begin{aligned}
& C_{\mathrm{O}}=\frac{k_{3 f} C_{\mathrm{H}_{2}}}{\left(2+\gamma_{3 b}\right) k_{1 b}}\left(\frac{k_{1 f}}{k_{4 f} C_{\mathrm{M}}}-1\right), \\
& C_{\mathrm{OH}}=\left[\frac{k_{3 f} k_{8 f}}{k_{1 b} k_{8 b}\left(2+\gamma_{3 b}\right)}\left(\frac{k_{1 f}}{k_{4 f} C_{\mathrm{M}}}-1\right)\right]^{1 / 2} C_{\mathrm{H}_{2} \mathrm{O} C_{\mathrm{H}_{2}}^{1 / 2},}^{1 / 2} \\
& C_{\mathrm{H}}=\left[\frac{k_{3 f}^{3} k_{8 f}}{k_{1 b} k_{8 b}\left(2+\gamma_{3 b}\right)^{3}}\left(\frac{k_{1 f}}{k_{4 f} C_{\mathrm{M}}}-1\right)\right]^{1 / 2} \frac{C_{\mathrm{H}_{2} \mathrm{O}}^{1 / 2} C_{\mathrm{H}_{2}}^{3 / 2}}{k_{4 f} C_{\mathrm{M}} C_{\mathrm{O}_{2}}},
\end{aligned}
$$


and

$C_{\mathrm{HO}_{2}}=\frac{k_{3 f} C_{\mathrm{H}_{2}}}{k_{7 f}\left(2+\gamma_{3 b}\right)}$.

These simplified expressions become accurate for very small values of $\mathrm{C}_{\mathrm{H}_{2}}$, as occurs for instance downstream from the reaction zone, where (A.15) and (A.16) are seen to describe accurately the slow decay of the $\mathrm{O}$ and $\mathrm{OH}$ radicals, as shown by the dot-dashed curves in Fig. A.2.

Clearly, the above equations can be also obtained as the limiting forms of (A.6)-(A.9) for $C_{\mathrm{H}_{2}} \ll 1$, when $\gamma_{8 f}-1 \ll 1, f \ll$ 1 , and, according to (A.10) and (A.11), $\tilde{G}=2+\gamma_{3 b}$ and $\tilde{H}=$ $\left\{\gamma_{8 b}\left[k_{1 f} /\left(k_{4 f} C_{\mathrm{M}}\right)-1\right] \tilde{G} / f\right\}^{1 / 2}$. The need for the 8-step description of $\mathrm{OH}$ is apparent from Eq. (A.16), which becomes singular if reaction 8 is deleted from the mechanism. The intricacy of the algebra is illustrated by the observations that (A.15), which differs from (28), does not involve any rate parameters of reaction 8 , even though that reaction and its rate parameters had to be included in its derivation, and that in all four denominators, the factor $2+\gamma_{3 b}$ differs from $1+\gamma_{3 b}$, the corresponding small- $f$ limit of $G$ in the 7-step mechanism.

Burning-velocity results can be derived from the 8-step mechanism that are quite similar to those obtained from the 7-step mechanism. In particular, agreements much like those seen in Fig. 8 are obtained. The analog of the approximation $H=1$ for the 7-step mechanism is the formula for $\tilde{H}$ given in the preceding paragraph for the 8-step mechanism, and it leads to roughly comparable agreements. Since the one-step approximation with $H=1$ derived from the 7-step mechanism yields good results that are simpler than those of the 8-step mechanism, it qualifies as a better theory for the overall reaction rate.

\section{References}

[1] F.A. Williams, J. Loss Prevent. Process Indust. 21 (2008) 131-135.

[2] J.B. Bell, M.S. Day, I.G. Shepherd, M. Johnson, R.K. Cheng, J.F. Grcar, V.E. Beckner, M.J. Lijewski, Proc. Natl. Acad. Sci. USA 102 (2005) 10006-10011.

[3] G. Dixon-Lewis, Philos. Trans. R. Soc. London A 292 (1979) 45-99.

[4] G. Balakrishnan, M.D. Smooke, F.A. Williams, Combust. Flame 102 (1995) 329340.

[5] L. He, P. Clavin, Combust. Flame 93 (1993) 391-407.

[6] F. Mauss, N. Peters, B. Rogg, F.A. Williams, in: N. Peters, B. Rogg (Eds.), Reduced Kinetic Mechanisms for Applications in Combustion Systems, Springer-Verlag, Heidelberg, 1993, pp. 29-43.

[7] K. Seshadri, N. Peters, F.A. Williams, Combust. Flame 96 (1994) 407-427.

[8] Available at: http://maemail.ucsd.edu/combustion/cermech.

[9] P. Saxena, F.A. Williams, Combust. Flame 145 (2006) 316-323.

[10] Cosilab Collection, Version 2.0.7, Rotexo-Softpredict-Cosilab GmbH \& Co. KG, Bad Zwischenahn, Germany, http://www.SoftPredict.com, 2007.

[11] D.R. Dowdy, D.B. Smith, S.C. Taylor, A. Williams, Proc. Combust. Inst. 23 (1990) 325-332.

[12] F.N. Egolfopoulos, C.K. Law, Proc. Combust. Inst. 23 (1990) 333-340.

[13] O.C. Kwon, G.M. Faeth, Combust. Flame 124 (2001) 590-610.

[14] H. Bongers, L.P.H. de Goey, Combust. Sci. Technol. 175 (2003) 1915-1928.

[15] F.A. Williams, J.F. Grcar, Proc. Combust. Inst. 32 (2008), in press.

[16] R.G. Gilbert, K. Luther, J. Troe, Ber. Bursenges. Phys. Chem. 87 (1983) 169177.

[17] J. Troe, Proc. Combust. Inst. 28 (2000) 1463-1469.

[18] G. del Álamo, F.A. Williams, A.L. Sánchez, Combust. Sci. Technol. 176 (2004) 1599-1626.

[19] A. Liñán, F.A. Williams, Fundamental Aspects of Combustion, Oxford University Press, New York, 1993, pp. 24-30.

[20] W.C. Reynolds, The Element Potential Method for Chemical Equilibrium Analysis: Implementation in the Interactive Program STANJAN (technical report), Stanford University, Dept. of Mechanical Eng., 1986.

[21] M.D. Smooke, V. Giovangigli, in: M.D. Smooke (Ed.), Reduced Kinetic Mechanisms and Asymptotic Approximations for Methane-Air Flames, in: Lecture Notes in Physics, vol. 384, Springer-Verlag, Berlin, 1991, pp. 1-28.

[22] T. Mitani, F.A. Williams, Combust. Flame 39 (1980) 169-190. 